\title{
Characterization of the UDP-glycosyltransferase UGT72 Family in Poplar and Identification of Genes Involved in the Glycosylation of Monolignols
}

\author{
Nathanael Speeckaert ${ }^{1,+}$, Nassirou Mahamadou Adamou 1,2, + , Hadjara Amadou Hassane 1,2(D), \\ Fabien Baldacci-Cresp ${ }^{1} \mathbb{D}$, Adeline Mol $^{1}$, Geert Goeminne ${ }^{3,4}$, Wout Boerjan ${ }^{3,5} \mathbb{D}^{D}$, \\ Pierre Duez $^{6}{ }^{\mathbb{D}}$, Simon Hawkins ${ }^{7}$, Godfrey Neutelings ${ }^{7} \mathbb{D}$, Thomas Hoffmann ${ }^{8}{ }^{\mathbb{D}}$, \\ Wilfried Schwab ${ }^{8}(\mathbb{D})$, Mondher El Jaziri ${ }^{1}$, Marc Behr ${ }^{1,+}{ }^{(D)}$ and Marie Baucher ${ }^{1, *,+}$ \\ 1 Laboratoire de Biotechnologie Végétale, Université libre de Bruxelles (ULB), \\ Rue des Professeurs Jeener et Brachet 12,6041 Gosselies, Belgium; naspeeck@ulb.ac.be (N.S.); \\ nassirou.mahamadou.adamou@ulb.ac.be (N.M.A.); hadjara.amadou.hassane@ulb.ac.be (H.A.H.); \\ fabien.baldaccicresp@gmail.com (F.B.-C.); adelimol@ulb.ac.be (A.M.); jaziri@ulb.ac.be (M.E.J.); \\ marc.behr@ulb.ac.be (M.B.) \\ 2 Laboratoire de Biotechnologie Végétale et Amélioration des Plantes (LABAP), \\ Université Abdou Moumouni de Niamey, BP 10727 Niamey, Niger \\ 3 Department of Plant Biotechnology and Bioinformatics, Ghent University, 9052 Ghent, Belgium; \\ gegoe@psb.vib-ugent.be (G.G.); woboe@psb.vib-ugent.be (W.B.) \\ 4 VIB Metabolomics Core, 9052 Ghent, Belgium \\ 5 VIB Center for Plant Systems Biology, 9052 Ghent, Belgium \\ 6 Unit of Therapeutic Chemistry and Pharmacognosy, Université de Mons, 7000 Mons, Belgium; \\ pierre.duez@umons.ac.be \\ 7 Unité de Glycobiologie Structurale et Fonctionnelle, Univ. Lille, CNRS, UMR 8576-UGSF, \\ F-59000 Lille, France; simon.hawkins@univ-lille.fr (S.H.); godfrey.neutelings@univ-lille.fr (G.N.) \\ 8 Biotechnology of Natural Products, Technische Universität München, 85354 Freising, Germany; \\ thomas.hofmann@tum.de (T.H.); wilfried.schwab@tum.de (W.S.) \\ * Correspondence: mbaucher@ulb.ac.be \\ + These authors contributed equally to this work.
}

Received: 28 June 2020; Accepted: 13 July 2020; Published: 16 July 2020

\begin{abstract}
Monolignols are the building blocks for lignin polymerization in the apoplastic domain. Monolignol biosynthesis, transport, storage, glycosylation, and deglycosylation are the main biological processes partaking in their homeostasis. In Arabidopsis thaliana, members of the uridine diphosphate-dependent glucosyltransferases UGT72E and UGT72B subfamilies have been demonstrated to glycosylate monolignols. Here, the poplar UGT72 family, which is clustered into four groups, was characterized: Group 1 UGT72AZ1 and UGT72AZ2, homologs of Arabidopsis UGT72E1-3, as well as group 4 UGT72B37 and UGT72B39, homologs of Arabidopsis UGT72B1-3, glycosylate monolignols. In addition, promoter-GUS analyses indicated that poplar UGT72 members are expressed within vascular tissues. At the subcellular level, poplar UGT72s belonging to group 1 and group 4 were found to be associated with the nucleus and the endoplasmic reticulum. However, UGT72A2, belonging to group 2, was localized in bodies associated with chloroplasts, as well as possibly in chloroplasts. These results show a partial conservation of substrate recognition between Arabidopsis and poplar homologs, as well as divergent functions between different groups of the UGT72 family, for which the substrates remain unknown.
\end{abstract}

Keywords: UGT72 family; poplar; monolignol glucosides; lignin; vascular tissues; gene expression; glycosyltransferases 


\section{Introduction}

Glycosyltransferases (GT) (EC 2.4.x.y) are defined as enzymes that utilize an activated donor sugar substrate that contains a (substituted) phosphate leaving group [1]. Glycosylation can modify the biochemical properties and/or the subcellular localization of a wide range of substrates, such as proteins, lipids, hormones, or phenylpropanoid compounds [2,3]. Based on available genome sequences, GT are ubiquitous enzymes, and 110 families have been identified so far, from which 42 are present in both model plants Arabidopsis thaliana and Populus trichocarpa (http://www.cazy.org/ GlycosylTransferases.html [4]). GT1, comprising the uridine diphosphate glycosyltransferases (UGT) that use uridine diphosphate (UDP)-activated sugars as donor molecules, is the largest GT family in plant [5]. The number of UGT has been estimated to be 122 (out of 497 GT) in A. thaliana and 281 (out of 788 GT) in P. trichocarpa [4]. UGT (E.C. 2.4.1.x) share a conserved motif, the Plant Secondary Product Glycosyltransferase (PSPG) box, consisting of 44 aa at the C-terminal part of the protein and which is involved in the binding of the UDP-sugar molecule [6]. UGTs glycosylate a large array of secondary metabolites, such as terpenoids, alkaloids, steroids, and flavonoids, as well as phenylpropanoids $[7,8]$.

Monolignols (or (hydroxyl)cinnamyl alcohols), i.e., p-coumaryl alcohol, coniferyl alcohol, and sinapyl alcohol, are the main building blocks of lignin and (neo)lignans [9]. When glycosylated by UGT, in the presence of UDP-glucose, these phenylpropanoids give rise to $p$-coumaryl alcohol glucoside, coniferin, and syringin, respectively (Figure 1). Monolignol glucosides accumulate in vascular tissues, including phloem, cambial tissue, and differentiating xylem, of both conifers and angiosperms, and they can be incorporated into the lignin polymer, as shown by experiments following injection of radio-labeled glycosylated monolignols in stems [10-20].

Glycosylation of monolignols renders them less cytotoxic by reducing their reactivity [21]. Monolignol glucosides presumably serve as storage or transport forms of monolignols [22,23], and their occurrence in vacuoles has been demonstrated [24]. Monolignols have been shown to be transported through the plasma membrane and monolignol glucosides through the tonoplast in the presence of ATP, indicating that their transport is ensured by ATP-binding cassette-like transporters that carry them to the cell wall or the vacuole, respectively [25]. In accordance, a $p$-coumaryl alcohol plasma membrane transporter, AtABCG29, has been identified and characterized in Arabidopsis [26]. However, as predicted by Vermaas et al. [27] by molecular dynamics simulation, the transport of monolignols to the apoplastic space is likely to occur also by passive diffusion across the plasma membrane.

In Arabidopsis, several recombinant proteins belonging to the UGT72 family, including UGT72E1-3 [28,29], as well as UGT72B1 and UGT72B3 [30], have been found to be able to glucosylate in vitro cinnamic acids, cinnamaldehydes and cinnamyl alcohols (Figure 1) with different affinities. More precisely, UGT72E1-3 may 4-O glucosylate coniferaldehyde and sinapaldehyde, as well as coniferyl and sinapyl alcohols with different specificities. UGT72E2 and UGT72E3 also accept ferulic and sinapic acids as substrates [29]. UGT72B1 displayed an activity with $p$-coumaryl, coniferyl and dihydroconiferyl alcohols, coniferaldehyde, and p-coumaraldehyde, while UGT72B3 could glucosylate coniferaldehyde and sinapaldehyde [30]. In contrast, UGT72C1, UGT72D1, and UGT72B2 had no activity towards these substrates [28,30]. An accumulation of coniferin and syringin, as well as ferulic acid 4-O-glucoside and sinapic acid 4-O-glucoside, was measured in Arabidopsis overexpressing UGT72E genes, with specificities depending on the gene overexpressed [31]. In contrast to lines with up- or down-regulation of UGT72E1-3, for which no lignin-associated phenotype and/or lignin modification (qualitative and quantitative) was reported, the ugt72b1 mutant was characterized by an ectopic lignification in floral stems, along with arrested growth [30]. When compared to the wild type (WT), both the $u g t 72 b 1$ mutant and transgenic Arabidopsis lines overexpressing UGT72B1 had an increase in coniferin [30]. As suggested by these authors, the origin of the higher amount of coniferin in the $u g t 72 b 1$ mutant is possibly related to the increased expression of both UGT72B3 and UGT72E2 that was detected in this mutant. 

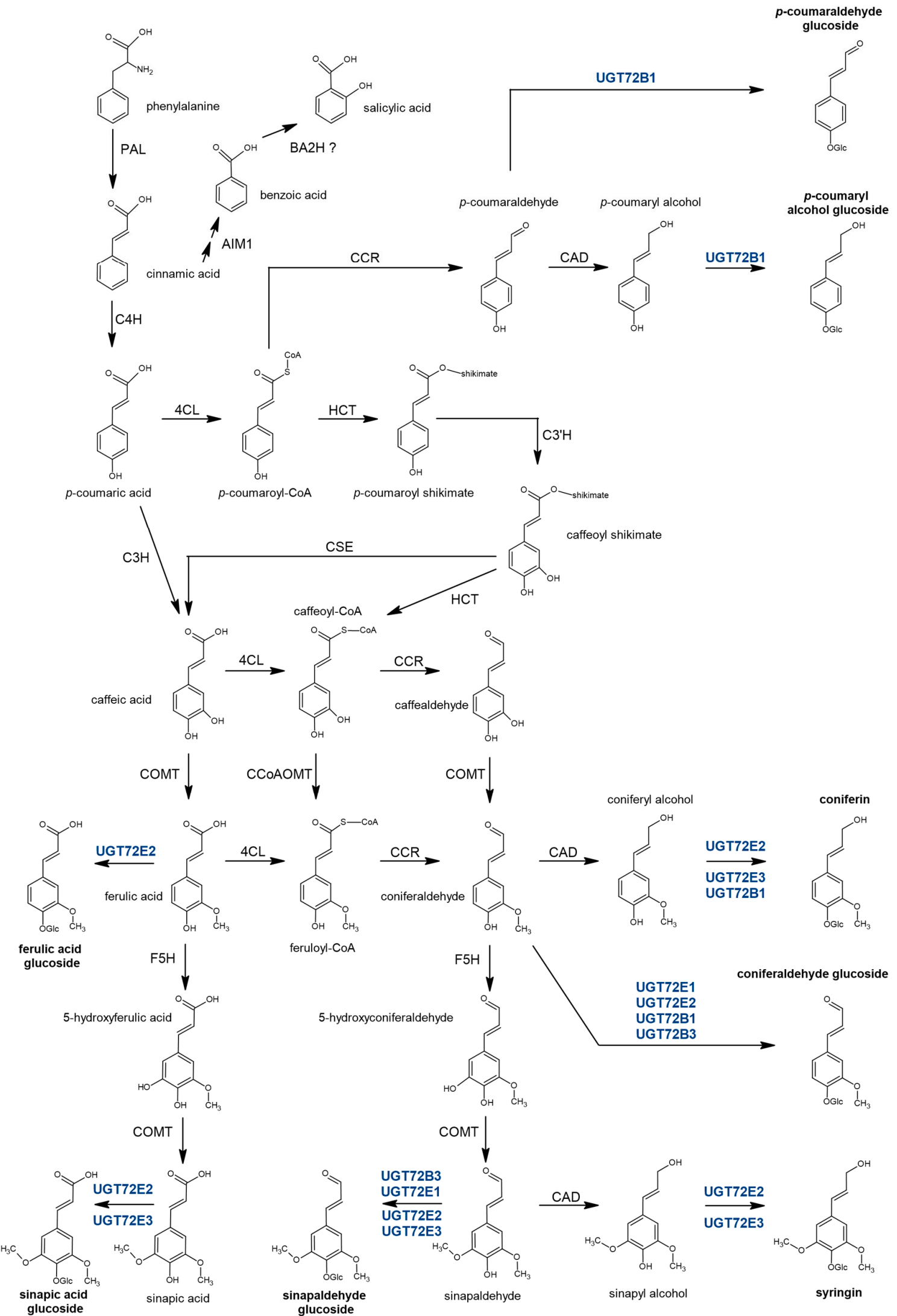

Figure 1. The monolignol biosynthetic pathway with associated glycosylated products and uridine diphosphate glucosyltransferases (UGT) catalyzing in vitro this glycosylation in Arabidopsis (indicated 
in blue), in presence of uridine diphosphate (UDP)-glucose (references within the text). UGT72E1 has been localized in the nucleus [32]. PAL-dependent salicylic acid biosynthetic pathway is also represented [33]. Glycosylated molecules are represented in bold. 4CL, 4-coumarate ligase; AIM1, abnormal inflorescence meristem 1; $\mathrm{BA} 2 \mathrm{H}$, benzoic acid 2-hydroxylase; $\mathrm{C} 3 \mathrm{H}, 4$-coumarate 3-hydroxylase; $\mathrm{C}^{\prime} \mathrm{H}$, 4-coumaroyl shikimate/quinate 3'-hydroxylase; $\mathrm{C} 4 \mathrm{H}$, cinnamate-4-hydroxylase; $\mathrm{CAD}$, cinnamyl alcohol dehydrogenase; CCoAOMT, caffeoyl-CoA 3-O-methyltransferase; CCR, cinnamoyl CoA reductase; COMT, caffeate $\mathrm{O}$-methyltransferase; CSE, caffeoyl shikimate esterase; $\mathrm{F} 5 \mathrm{H}$, ferulate 5-hydroxylase; HCT, hydroxycinnamoyl-CoA:shikimate/quinate hydroxycinnamoyltransferase; PAL, phenylalanine ammonia-lyase.

Overall, these reports indicate that different recombinant glycosyltransferases from the Arabidopsis UGT72 family have different substrate specificities in vitro, suggesting that they are all involved in a complex regulatory network required for adequate maintenance of the monolignol/monolignol glucoside pool, as well as that of their acid or aldehyde precursors (Figure 1).

The aim of the present study was to characterize the UGT72 family in poplar (P. tremula $\times$ P. alba clone INRA 717-1B4), a woody perennial plant, to identify which members are involved in the glycosylation of monolignols. Our results showed that poplar UGT72 family members clustered into four groups. Enzymatic activities of each member, tested with 11 different phenylpropanoid substrates, as well as analysis of glycosylated monolignols in transgenic poplars overexpressing the corresponding genes, allowed the identification of 4 poplar UGT72s glycosylating monolignols, including members of group 1 (UGT72AZ1 and UGT72AZ2) and group 4 (UGT72B37 and UGT72B39). The poplar UGT72s belonging to group 2 and 3 had no activity towards the tested substrates. Analysis of promoter-GUS expression profiles showed expression in vascular tissues for all genes analyzed. Finally, differences in subcellular localization were found for the poplar UGT72s. Altogether, these data suggest different functions for members of the UGT72 family in poplar.

\section{Results}

\subsection{Cloning of the Full Length cDNA of Poplar UGT72 Genes}

Ten sequences homologous to Arabidopsis UGT72s were identified in the P. trichocarpa genome sequence. Primers to obtain P. tremula $\times$ P. alba orthologous sequences were designed for 8 of these genes (Table S1). Potri.007G029800 was not cloned because its coding sequence was $99 \%$ identical to that of Potri.007G030300 (UGT72AZ1), and Potri.014G041900 was not cloned as it has a truncated PSPG motif, and the catalytically active His at the N-terminus is missing (Figure S1). None of these genes had introns. The P. tremula $\times$ P. alba UGT72 genes were named UGT72A2, UGT72AZ1, UGT72AZ2, UGT72B36, UGT72B37, UGT72B38, UGT72B39, and UGT72BB1, according to the UGT Nomenclature Committee (https://prime.vetmed.wsu.edu/resources/udp-glucuronsyltransferase-homepage). An alignment of the different deduced polypeptide sequences of UGT72 members of P. tremula $\times$ P. alba and Arabidopsis shows the PSPG domain and conserved key residues in these sequences (Figure S1). The percentages of identity between poplar UGT72s and their Arabidopsis homologs is given in Table S2, and their respective polypeptide length, predicted $\mathrm{pI}$, and predicted molecular weight in Table S3.

As shown in Figure 2, the analysis of the phylogenetic relationships between UGT72s of Arabidopsis and poplar revealed that the proteins clustered into 4 different groups. The first group comprises the Arabidopsis UGT72E1-E3 and UGT72C1, as well as the poplar UGT72AZ1-AZ2. The second group is made up of the Arabidopsis UGT72D1-D2P and the poplar UGT72A2. The third group contains UGT72BB1 with no Arabidopsis homolog, and the fourth group includes UGT72B members from both Arabidopsis (B1-B3) and poplar (B36-B39). 


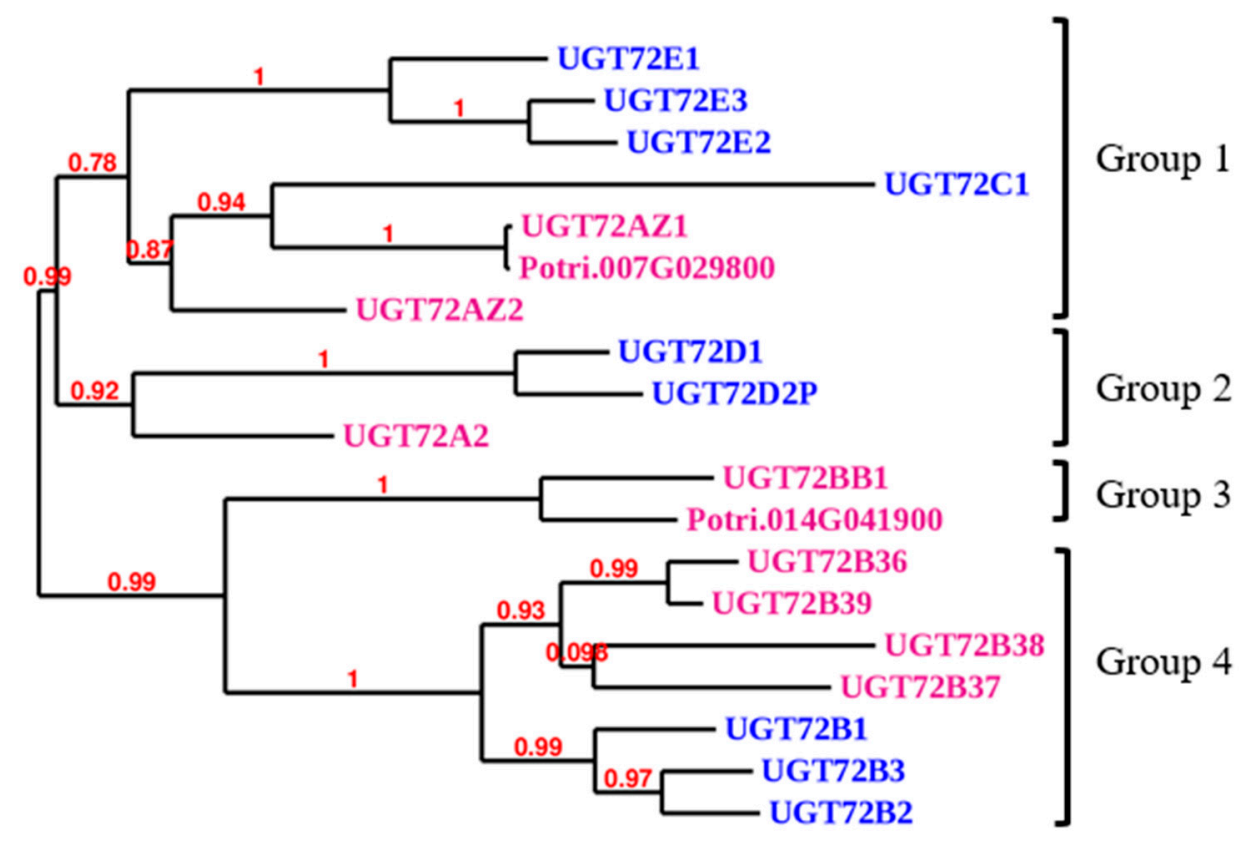

0.3

Figure 2. Phylogenetic tree of UGT72s from Arabidopsis and poplar. This tree was generated by the approximate likelihood-ratio test method. Arabidopsis and poplar polypeptides are shown in blue (as in Figure 1) and magenta, respectively. Scale bar: expected numbers of amino acid substitutions per site. Protein alignment is given in Figure S1.

\subsection{Recombinant UGT72B37 and UGT72B39 Proteins Glycosylate Monolignols}

The 8 poplar UGT72s were cloned in the pGEX-4T-1 expression vector containing an N-terminal glutathione S-transferase (GST-fusion) tag. After production in E. coli and purification, recombinant proteins were tested using 11 substrates of the phenylpropanoid/monolignol pathway (Figure 1) using UDP-glucose as sugar donor. As shown in Table 1, as well as in Figure 3 and Figure S2, recombinant UGT72AZ2, UGT72B37, and UGT72B39 recognized at least one substrate in vitro, whereas no activity towards any of the tested substrates was detected for the 5 other UGT72s. UGT72B37 (group 4) showed a broad substrate specificity as it could glycosylate $p$-coumaraldehyde, coniferaldehyde, sinapaldehyde, coniferyl alcohol, and sinapyl alcohol (Figure 3), while UGT72B39 (group 4) only glycosylated coniferyl alcohol (Figure S2), and UGT72AZ2 (group 1) glycosylated ferulic and sinapic acids (Figure S2).

Table 1. Poplar UGT72s activity towards different phenylpropanoids.

\begin{tabular}{lccc}
\hline Substrates & $\begin{array}{l}\text { UGT72AZ2 } \\
\text { (Group 1) }\end{array}$ & $\begin{array}{l}\text { UGT72B37 } \\
\text { (Group 4) }\end{array}$ & $\begin{array}{l}\text { UGT72B39 } \\
\text { (Group 4) }\end{array}$ \\
\hline$p$-coumaraldehyde & - & + & - \\
coniferaldehyde & - & + & - \\
sinapaldehyde & - & + & - \\
coniferyl alcohol & - & + & + \\
sinapyl alcohol & - & + & - \\
trans-cinnamic acid & - & - & - \\
$p$-coumaric acid & - & - & - \\
caffeic acid & - & - & - \\
ferulic acid & + & - & - \\
sinapic acid & + & - & - \\
salicylic acid & - & - & - \\
\hline
\end{tabular}

- no activity detected; + , activity detected. 

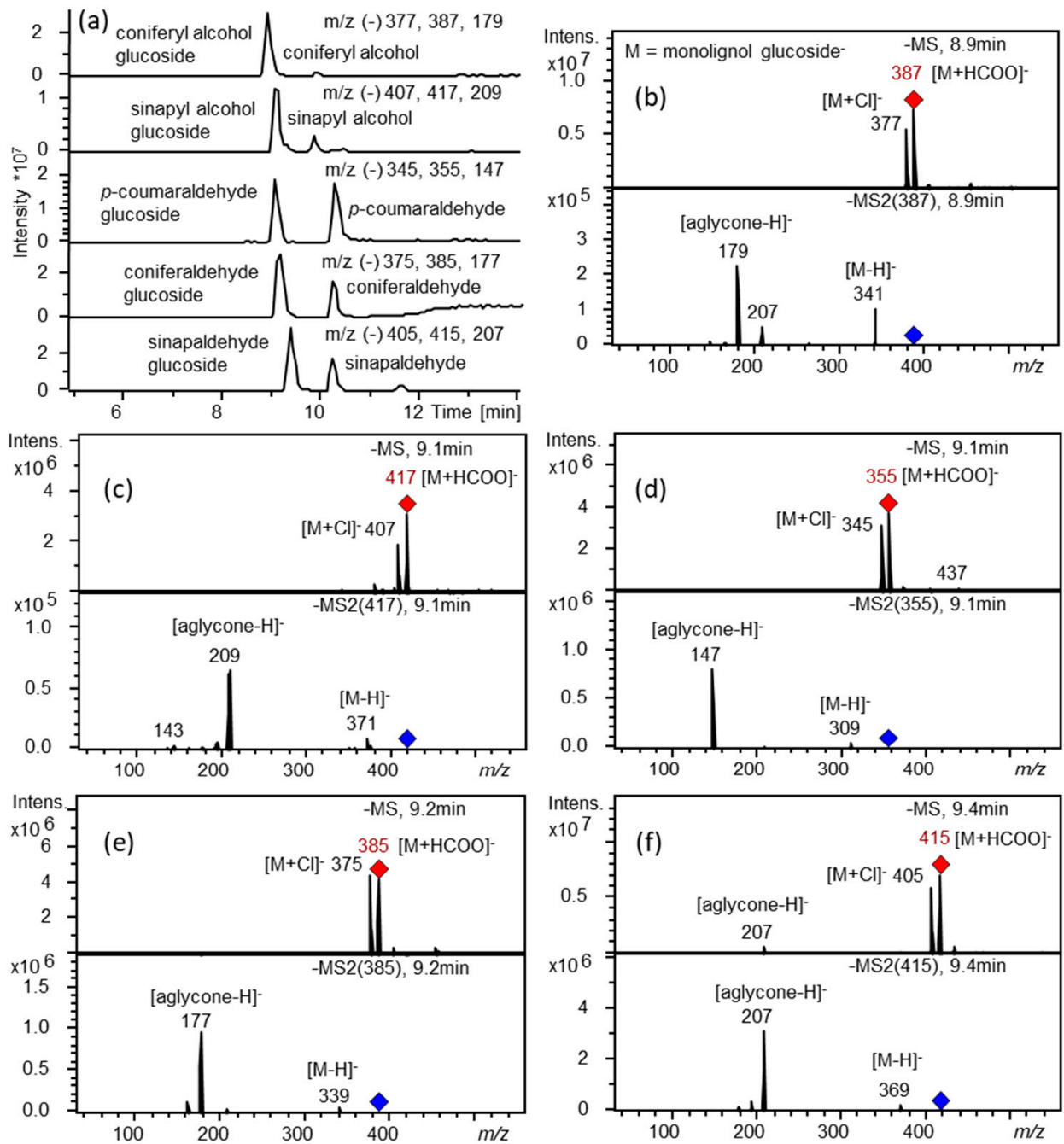

Figure 3. LC-MS analysis of UGT72B37 enzymatic reaction products. Extracted ion chromatograms (a), mass spectra (MS in red) and product ion spectra (MS2 in blue) of compounds formed by UGT72B37 with coniferyl alcohol (b), sinapyl alcohol (c), p-coumaraldehyde (d), coniferaldehyde (e), and sinapaldehyde

(f) as substrates. UDP-glucose was used as sugar donor. M, monolignol glucoside.

\subsection{Accumulation of Monolignol Glycosides in Transgenic Poplar Overexpressing UGT72s}

Transgenic overexpression poplar lines were produced for all members of the UGT72 family, except for UGT72B38, for which no plants could be regenerated. Overexpression of each UGT72 in these lines was confirmed by RT-qPCR on leaves of plants cultivated for 4 months in soil. As shown in Figure S3, the level of overexpression was variable for the different constructs, being about 1000-8000-fold for 35S-UGT72AZ1 and 35S-UGT72AZ2 lines (group 1), 2500- to 4500-fold for the 35S-UGT72BB1 lines (group 3), and 200-900-fold in 35S-UGT72A2 lines (group 2), whereas it reached 10- to maximum 70-fold in the 35S-UGT72B36, 35S-UGT72B37, and 35S-UGT72B39 (group 4) lines. The differences between the overexpression levels among the different UGT72 genes might be related to the endogenous level of expression of these genes in leaves, which seems inversely related with the fold-increased level. Indeed, in silico analysis of gene expression in different tissues of 1-year-old P. trichocarpa based on the data published by Shi and colleagues [34] shows that UGT72AZ1-2 (group 1) and UGT72BB1 (group 3) are expressed at low levels in leaves (transcript abundance between 2 and 8), whereas UGT72A2 (group 2) shows an intermediate expression (transcript abundance of approximately 150) compared to UGT72B36-39 (group 4), which are highly expressed (transcript abundance between 150 and 1000) (Figure S4A). 
Examination of the transgenic plants revealed that no phenotypical (at the whole plant level), nor anatomical differences (in transversal stem cross sections stained with phloroglucinol) could be observed in any of the different poplar UGT72 overexpressing lines. To further characterize these lines, we investigated the profiles of glycosylated phenolics in leaf extracts in order to identify the in vivo acceptor substrates. LC-MS analysis (Figure 4) indicated that the lines overexpressing the group 1 genes accumulated coniferin and syringin (UGT72AZ1), or just coniferin (UGT72AZ2). Traces of coniferin were detected in the WT, while syringin was below the detection level. Transgenic lines overexpressing group 2 or group 4 UGT72 genes did not accumulate detectable amounts of both glycosides using a HPLC-UV system (no plant material was available for group 3).

To assess whether the observed increase in the quantity of coniferin and syringin levels in leaves was accompanied by modifications in lignin levels, we analyzed stem wood, stem bark, and root samples from 35S-UGT72AZ1 and 35S-UGT72AZ2 lines. Our results indicated that lignin content, as measured by the acetyl bromide method, was not affected in any of the samples from these lines (Figure S5).

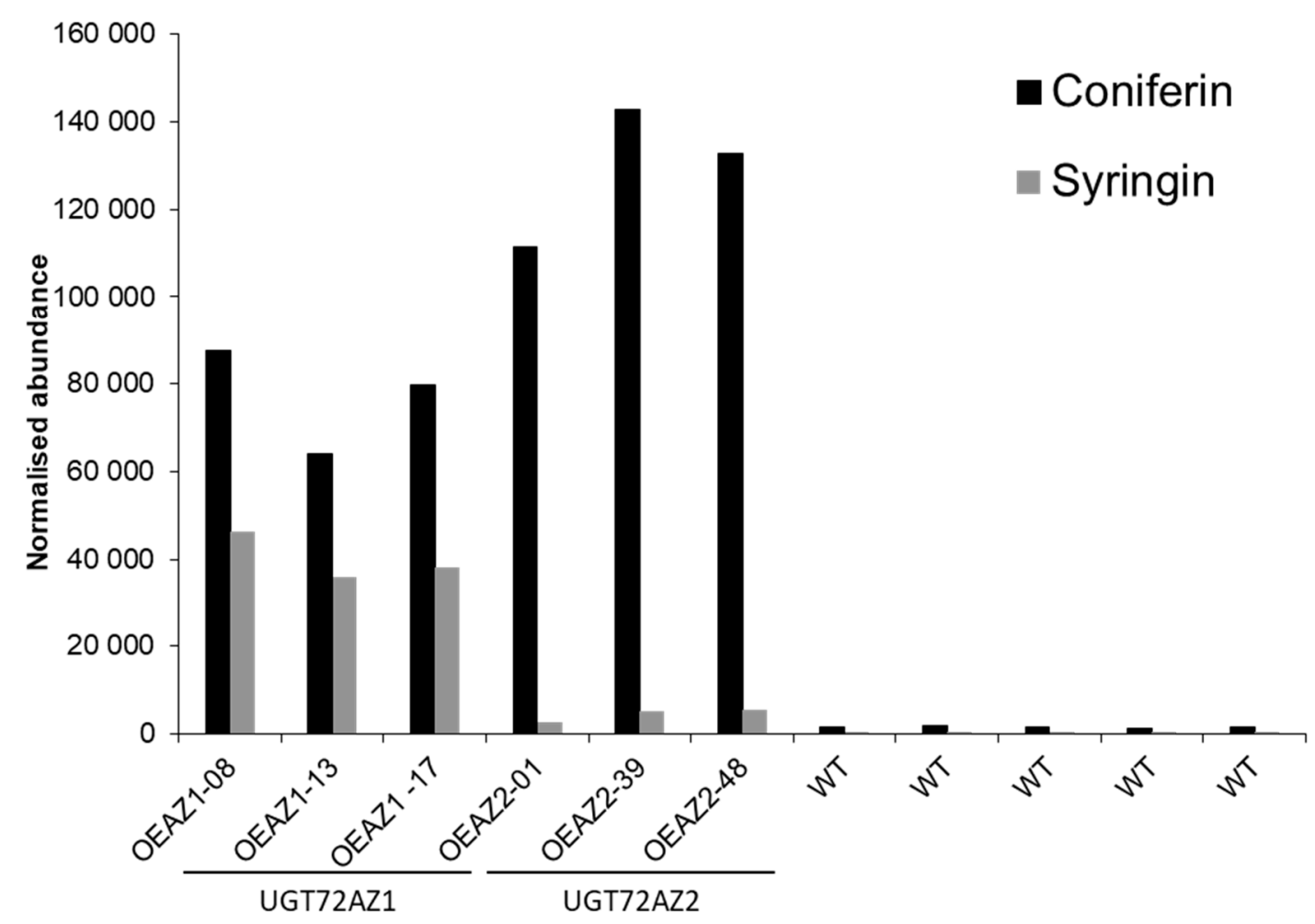

Figure 4. LC-MS analysis of coniferin and syringin in leaf of 4-month-old wild type (WT), UGT72AZ1, and UGT72AZ2 overexpressing lines grown in the greenhouse. Measures were made in 5 independent WT and in 3 independent transgenic lines for each construct. This analysis was repeated with HPLC-UV with similar results.

\subsection{UGT72s Expression Profiles in Poplar}

To further characterize the different poplar UGT72 genes, their expression profile was analyzed by RT-qPCR in different parts of the stem and the root of 4-month-old greenhouse grown WT poplar. Primary vascular tissues (primary VT) correspond to the first $2 \mathrm{~cm}$ below the apex. Secondary vascular tissues (secondary VT) were sampled $50 \mathrm{~cm}$ below the apex and separated into bark and wood (entitled phloem and xylem in Figure 5, respectively). Young root with primary VT and old root with secondary VT ( $\sim \mathrm{cm}$ diameter) were also investigated. As shown in Figure 5, expression of the eight genes was detected in all samples but at different levels. UGT72AZ1 was principally expressed in the phloem of the stem, UGT72AZ2 in young roots, UGT72A2 in young stems, UGT72BB1 in the phloem of the stem 
and in roots (thus possibly in the phloem of the root), and UGT72B36 in xylem of the stem and in old roots (thus possibly in the xylem of root), while UGT72B37 was expressed in all parts with the highest level in stem secondary xylem, UGT72B38 in young stems and roots (thus possibly in primary VT), and UGT72B39 in the xylem of the stem and in young roots (probably in the xylem of the root).

UGT72AZ1

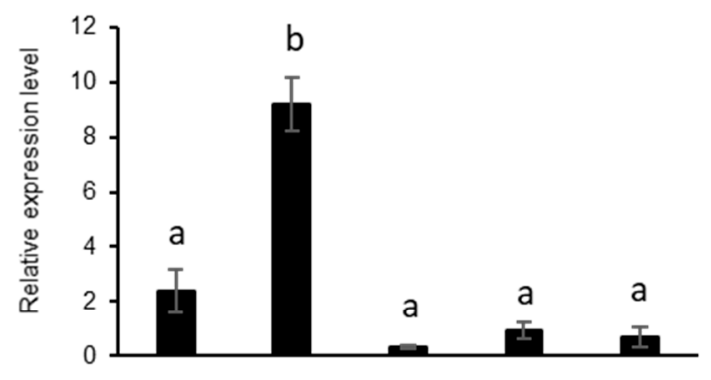

UGT72A2
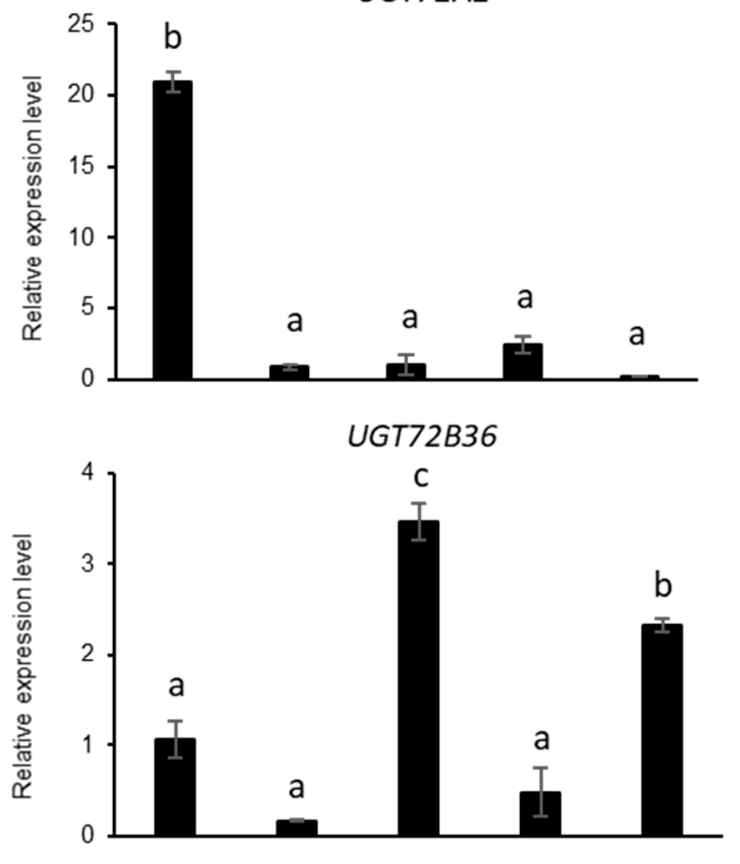

UGT72B38

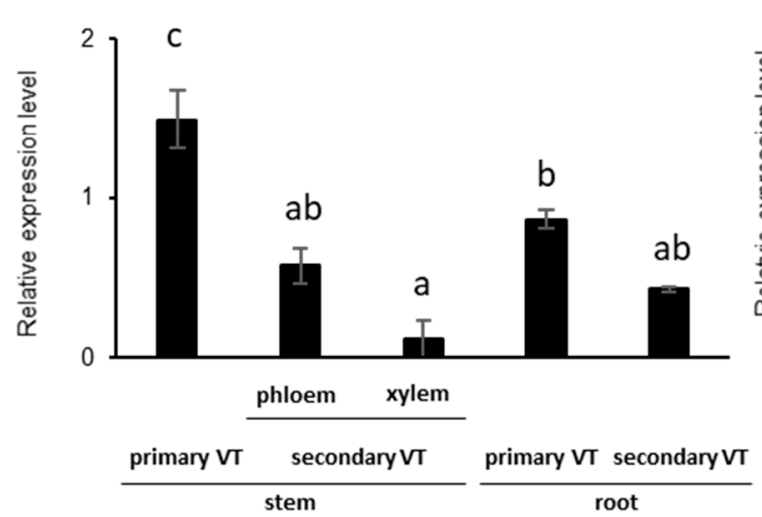

UGT72AZ2

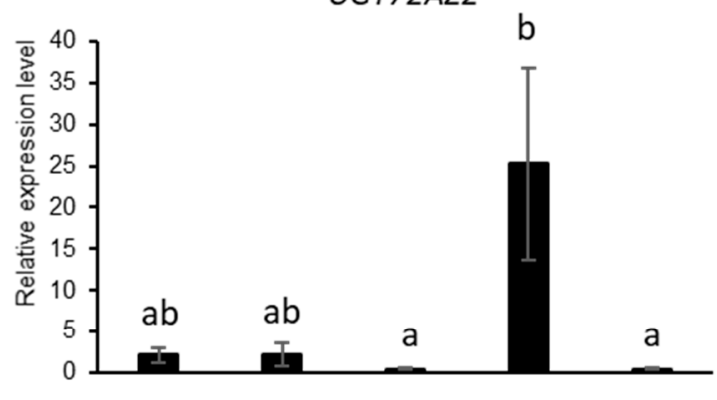

UGT72BB1
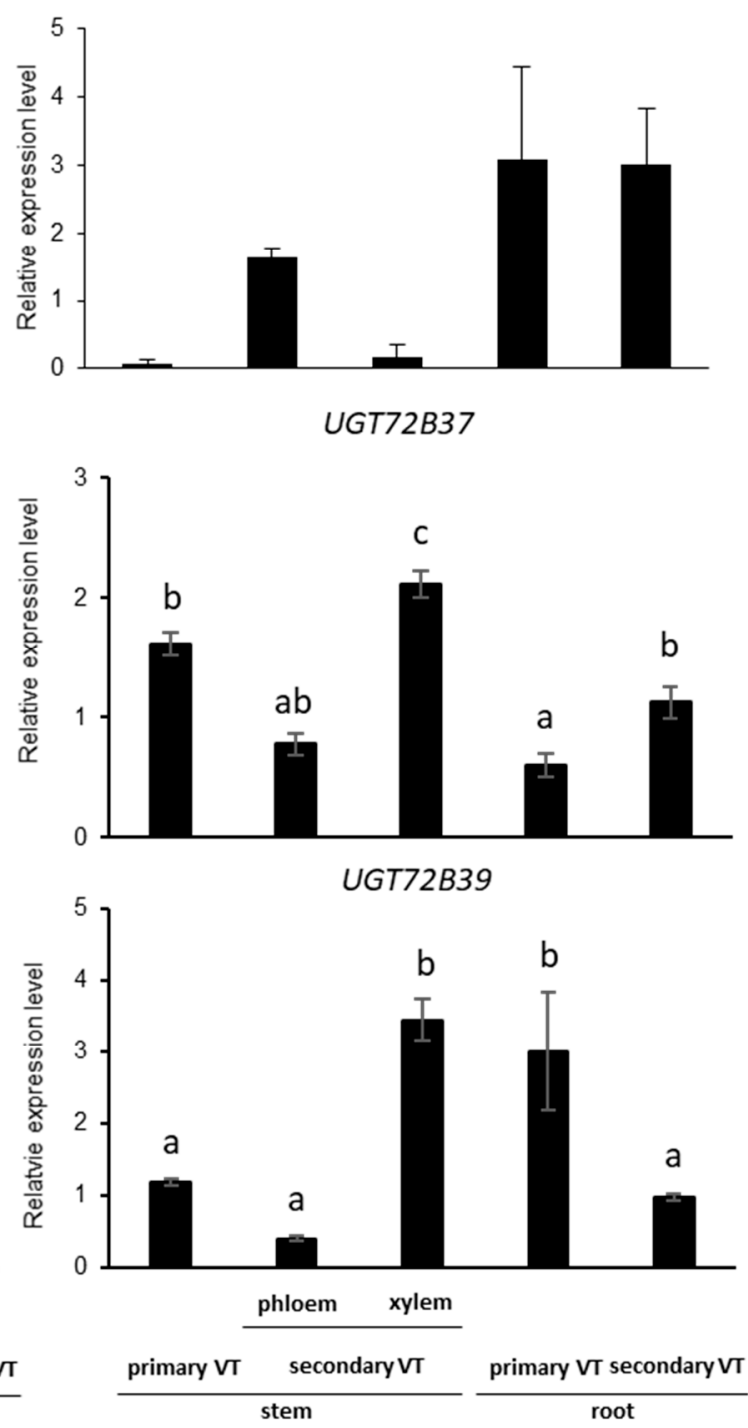

Figure 5. RT-qPCR expression profile of UGT72s in different parts of 4-month-old poplar trees grown in the greenhouse. Normalized relative expressions are means of 3 biological replicates $( \pm S E)$. Significantly different values $(p<0.05)$ are represented by different letters (ANOVA and Tukey post hoc test). VT, vascular tissue. 


\subsection{UGT72s Are Expressed within Vascular Tissues}

In order to confirm the expression data obtained by RT-qPCR and provide more detailed information about UGT72s spatial expression, histochemical GUS staining of $p U G T 72:: G U S$ transgenic poplar lines was investigated. Unfortunately, no plants could be regenerated for the pUGT72B38::GUS and $p U G T 72 B B 1:: G U S$ constructs. A focus was made on the organs where the expression measured by RT-qPCR of the respective genes was most pronounced (i.e., root for UGT72AZ2 and stem for UGT72A2, UGT72B36, UGT72B37, and UGT72B39). As shown in Figure 6 and Figure S6, the 6 UGT72 genes are expressed in vascular tissues with different specificities. UGT72AZ1 is expressed mainly in the phloem within the stem and leaf (Figure 6a and Figure S6b), UGT72AZ2 in the cortical region, as well as in the phloem and differentiating xylem of the root (Figure 6b), UGT72A2 (Figure 6c), and UGT72B39 (Figure 6f,i) in the primary xylem of stem and UGT72B36 (Figure 6d,g) and UGT72B37 (Figure 6e,h) within the stem xylem. These results are in accordance with the RT-qPCR results (Figure 5) and with RNA-Seq data obtained from different cross sections within vascular tissues of the stem of a 45-year-old P. tremula (Figure S4b, heatmap made with data from group 4 taken from the AspWood database [35]).

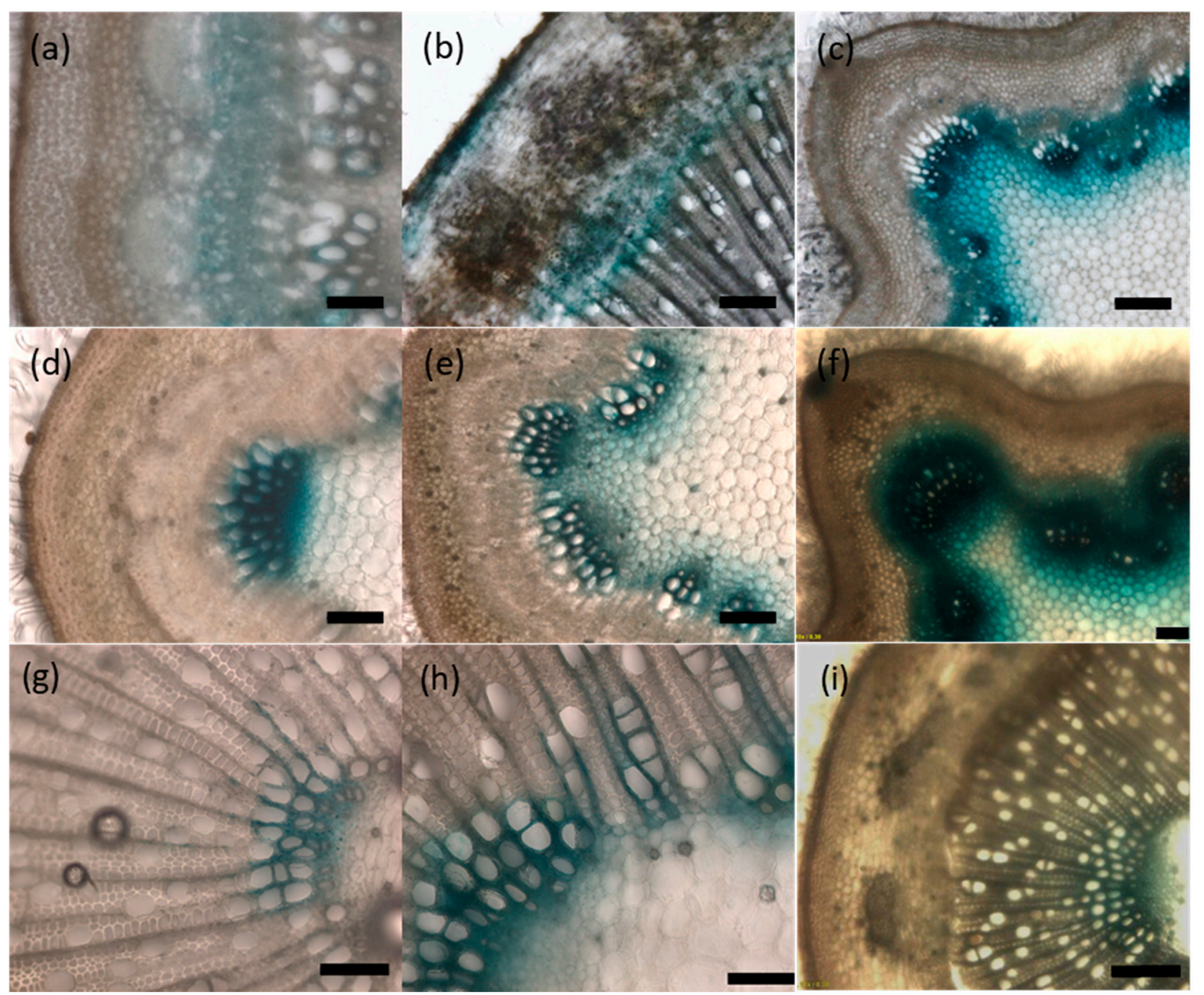

Figure 6. GUS expression patterns in tissues of 4-month-old poplar grown in the greenhouse. (a) pUGT72AZ1::GUS expression in the stem $10 \mathrm{~cm}$ below the apex. (b) pUGT72AZ2::GUS expression in $4 \mathrm{~mm}$ diameter root. (c) $p U G T 72 A 2::$ GUS expression in stem $5 \mathrm{~cm}$ below the apex. (d) $p U G T 72 B 36:: G U S$ expression in stem $10 \mathrm{~cm}$ below the apex. (e) $p U G T 72 B 37::$ GUS expression in stem $10 \mathrm{~cm}$ below the apex. (f) pUGT72B39::GUS expression in stem $5 \mathrm{~cm}$ below the apex. (g) pUGT72B36::GUS expression in stem $30 \mathrm{~cm}$ below the apex. (h) pUGT72B37::GUS expression in stem $30 \mathrm{~cm}$ below the apex. (i) $p U G T 72 B 39:: G U S$ expression in stem $30 \mathrm{~cm}$ below the apex. Scale $=100 \mu \mathrm{m}(\mathbf{a}, \mathbf{c}, \mathbf{f}, \mathbf{i}), 200 \mu \mathrm{m}(\mathbf{b}, \mathbf{d}, \mathbf{e}, \mathbf{g}, \mathbf{h})$. 
In silico UGT72s promoter analysis (Figure 7 and Tables S4 and S5) reveals the occurrence of cis-elements involved in the differentiation of vascular tissues, such as Secondary wall MYB-Responsive Element (SMRE), Secondary wall NAC-Binding Element (SNBE), R2R3MYB Responsive Element (AC-elements), and Tracheary-Element-Regulating cis-Element (TERE) [36,37]. SNBE were present in all promoters (from one element in $p U G T 72 B 39-B B 1$ to five elements in $p U G T 72 A 2 / A Z 2$ ). TERE is found in several genes related to xylem differentiation [38] and was detected only in $p U G T 72 B 36$. Several SMRE and AC-elements were detected in $p U G T 72 B B 1, p U G T 72 B 36, p U G T 72 B 37$, and $p U G T 72 B 39$, while $p U G T 72 B 38$, $p U G T 72 A Z 1$, and $p U G T 72 A 2$ displayed a single SMRE, and $p U G T 72 A Z 2$ did not exhibit any of these cis-elements. The presence of these cis-elements may explain the preferential expression of the poplar UGT72 genes in vascular tissues (Figures 5 and 6).

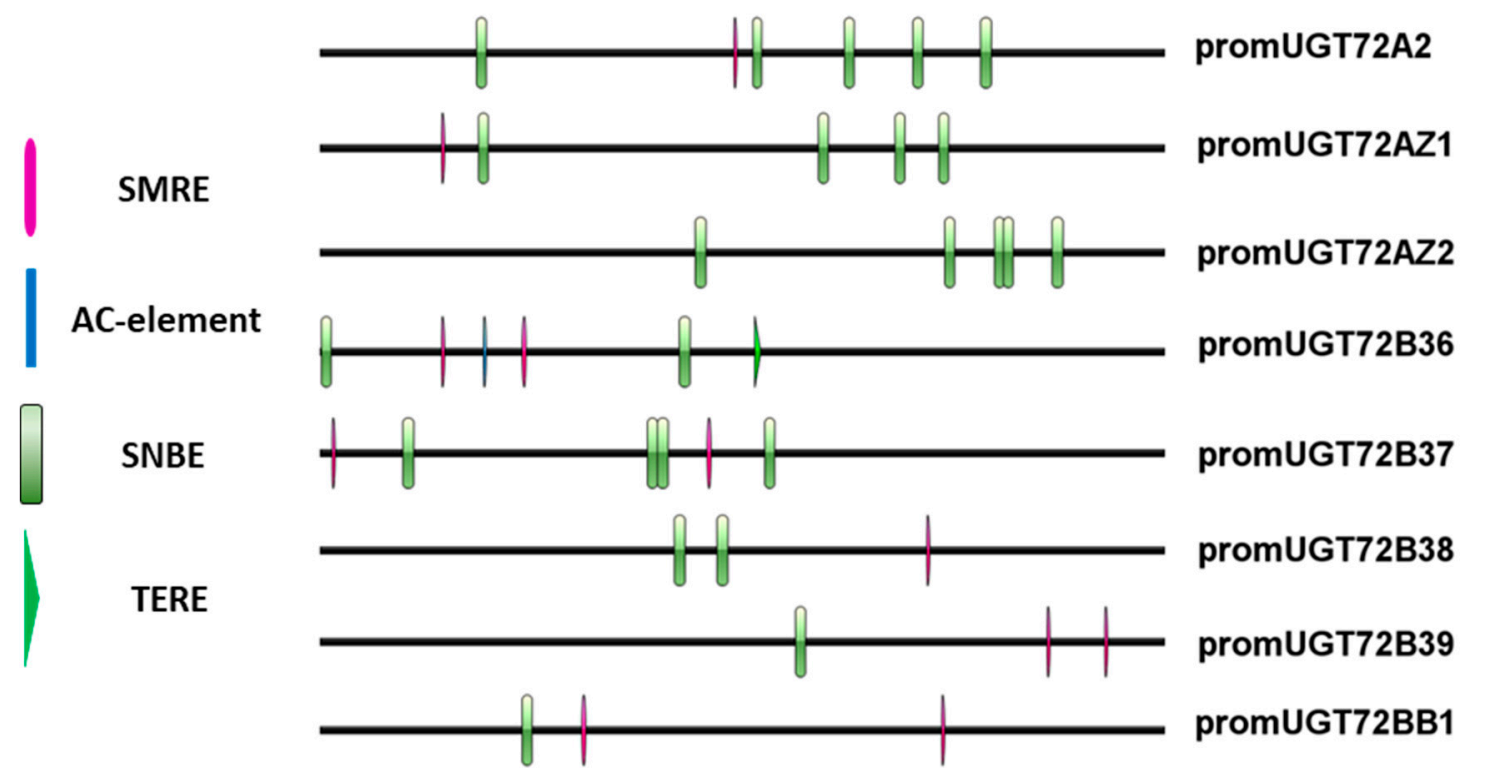

Figure 7. Secondary cell wall-related cis-elements in the promoters of poplar UGT72 members. SMRE, Secondary wall MYB-Responsive Element. SNBE, Secondary wall NAC-Binding Element. AC-element, R2R3MYB Responsive Element. TERE, Tracheary-Element-Regulating cis-Element. The cis-element sequences and the associated binding factors in Arabidopsis are listed in Table S4. The analysis was made on the 1500 bp upstream the coding sequences.

2.6. Proteins of Members of The Poplar UGT72 Family Preferentially Accumulate in Vascular Tissues

The immunolocalization of UGT72AZ1, UGT72AZ2 (group 1) and UGT72A2 (group 2), for which custom antibodies were designed, was investigated in P. tremula $\times P$. alba grown in the greenhouse (for antibody characterization, see Figure S7). Based on GUS histochemical staining and RT-qPCR results, immunolocalizations were performed in stem for UGT72AZ1 and UGT72A2 and in root for UG72AZ2. As shown in Figure 8, UGT72AZ1 was localized mainly in the phloem (Figure 8a), the cambium and the developing xylem of the stem, while UGT72AZ2 was located mainly in the cambium, as well as in the cortex of the root (Figure $8 \mathrm{~b}$ ). UGT72A2 was principally detected in the phloem but also in the differentiating xylem and in ray cells of the xylem, as well as in the pith (Figure 8c). These results indicate that UGT72AZ1, UGT72AZ2, and UGT72A2 seem to preferentially accumulate in vascular tissues, albeit with different patterns.

\subsection{Subcellular Localization of the Poplar UGT72s}

To investigate the subcellular localization of poplar UGT72 members, C-terminal GFP-fused UGTs were transiently expressed in tobacco leaf epidermis using agroinfiltration, together with an endoplasmic reticulum (ER) marker. As shown in Figure 9, five UGT72s (UGT72AZ1 and UGT72AZ2 from group 1 and UGT72B36, UGT72B37, and UGT72B39 from group 4) showed a defined ER and nuclear localization 
(Figure 9a-e). In contrast, UGT72A2 (group 2) was localized in bodies associated with the chloroplasts (Figure 9f). The UGT72A2 localization was further investigated in stable transgenic poplars harboring the same 35S::UGT72A2-GFP construct. In this case, the localization of UGT72A2-GFP was also observed in bodies associated with the chloroplasts and possibly within chloroplasts (Figure 9g).

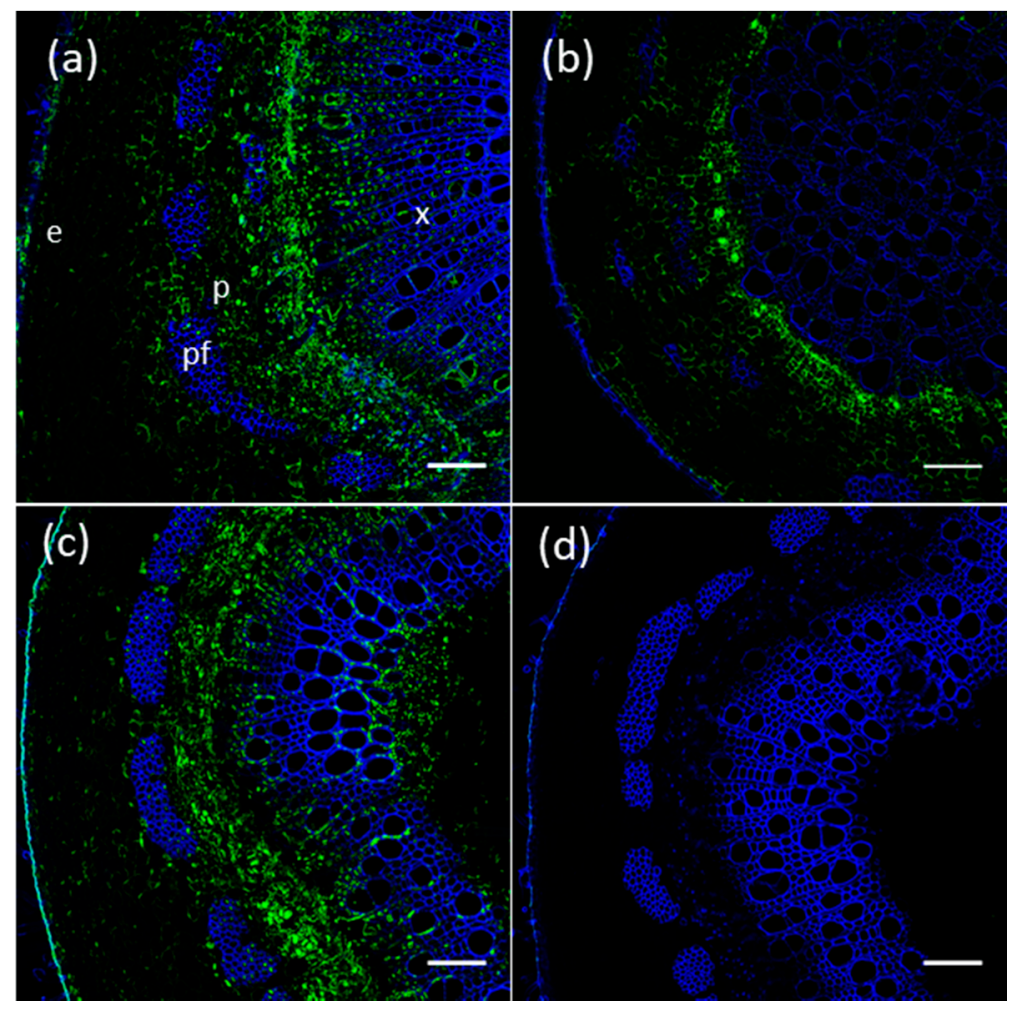

Figure 8. Immunolocalization of UGT72AZ1, UGT72AZ2, and UGT72A2 in transversal sections of 3-month-old WT poplar grown in the greenhouse. (a) UGT72AZ1 in the stem at $20 \mathrm{~cm}$ below the apex. (b) UGT72AZ2 in the root. (c) UGT72A2 in the stem at $10 \mathrm{~cm}$ below the apex. (d) Negative control in stem (no primary antibody). Alexa 488 signal corresponding to the UGT72 epitope is shown in green. Lignin autofluorescence is in blue. e, epidermis; $p$, phloem; pf, phloem fiber; $x$, xylem. Scale $=100 \mu \mathrm{m}$.

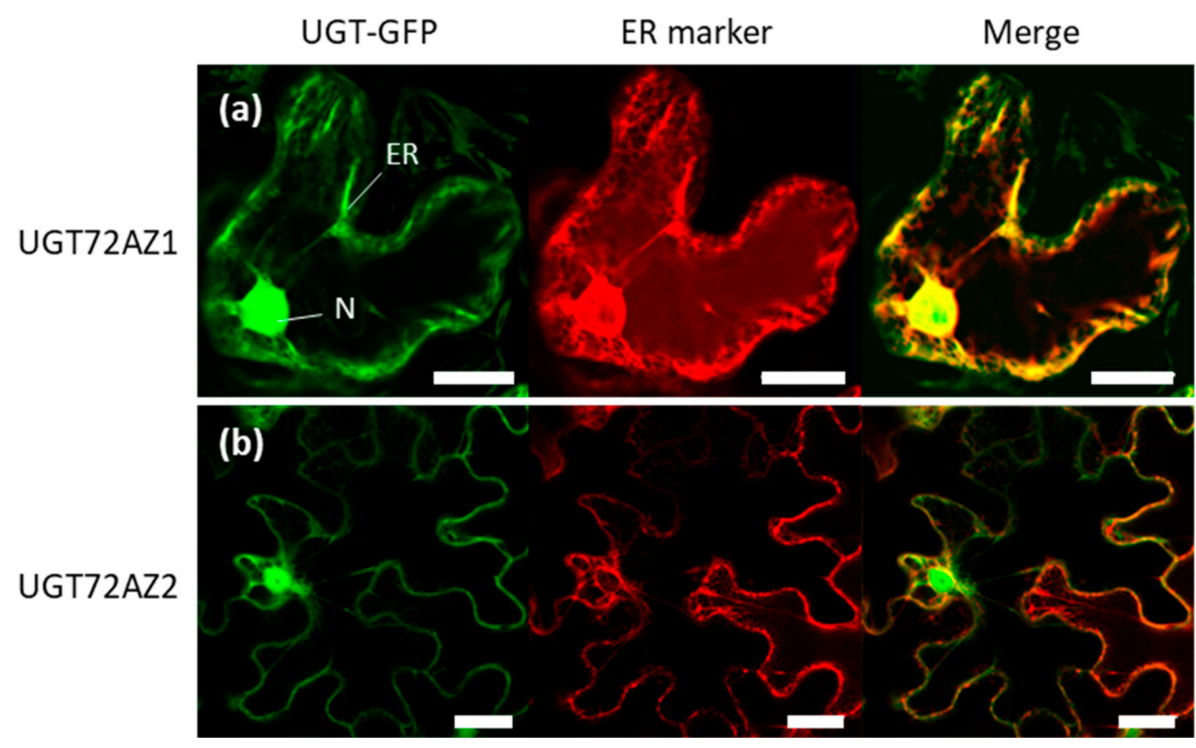

Figure 9. Cont. 

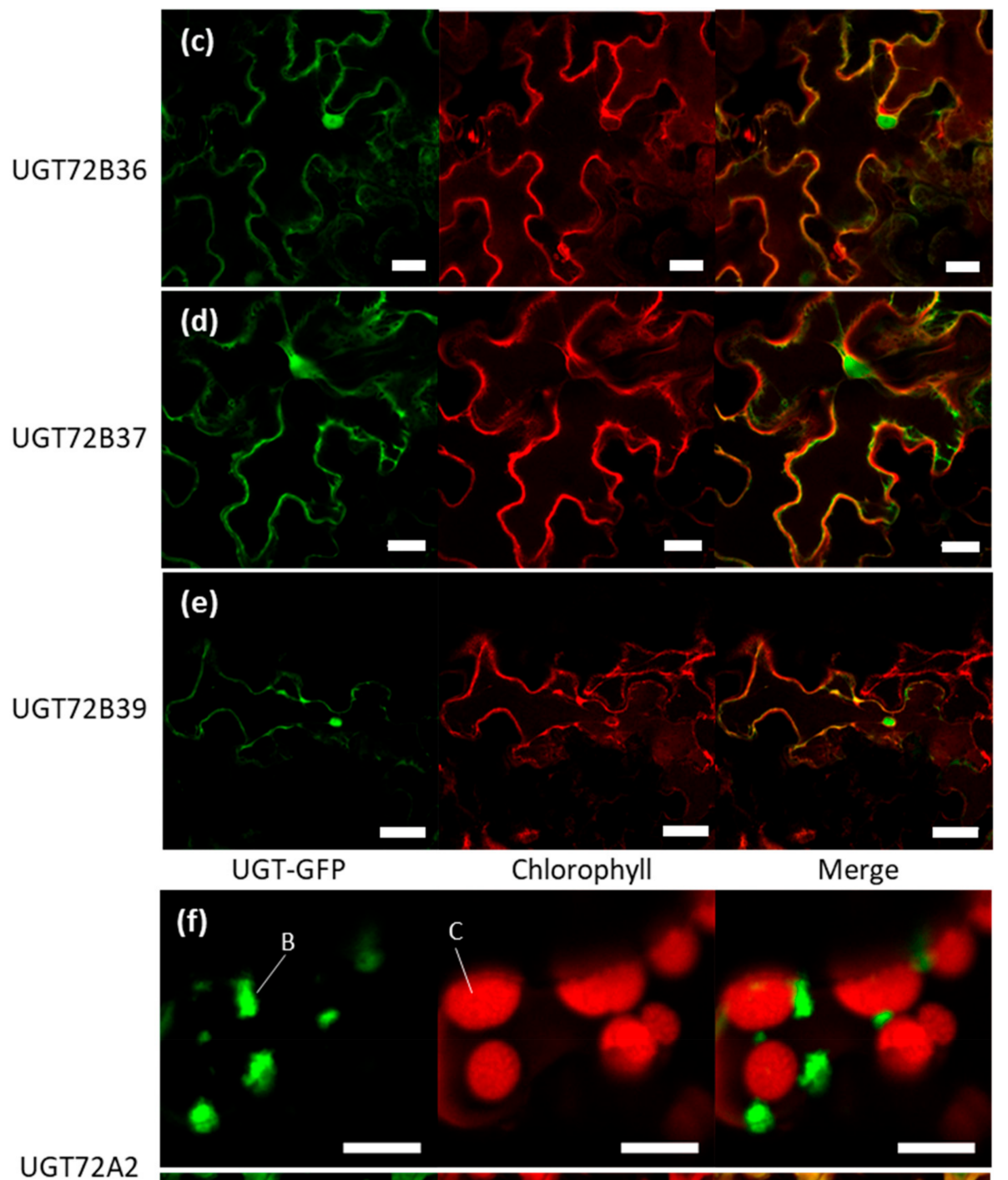

UGT72A2

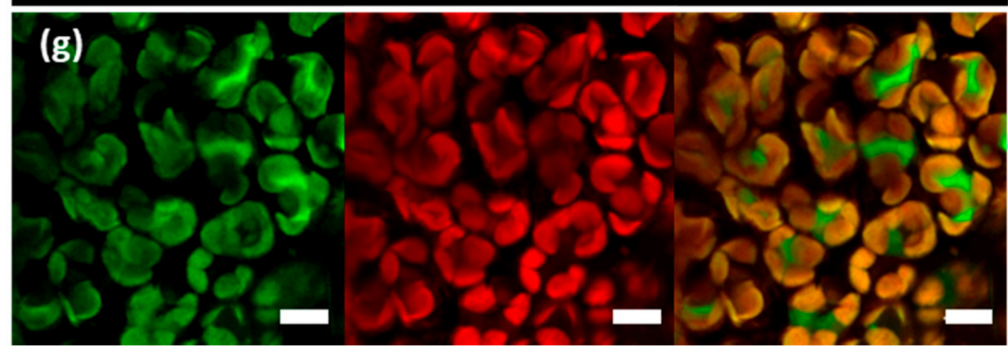

Figure 9. Subcellular localization of poplar UGT72 fused to GFP. (a-f) UGT72-GFP fusion proteins and 35S::AtWAK2-mCherry, as ER marker (a-e) in N. benthamiana leaf epidermal cells after agroinfiltration. UGT72A2-GFP (f) was localized in bodies associated to chloroplasts (red fluorescence). (g) Stable transgenic poplar line expressing the same 35S::UGT72A2-GFP construct. UGT72A2 was localized in in bodies associated with chloroplasts and possibly in chloroplasts. Images are representative of biological triplicates. B, bodies associated with chloroplast; C, chloroplast; ER, endoplasmic reticulum; $\mathrm{N}$, nucleus. Scale $=20 \mu \mathrm{m}(\mathbf{a}), 35 \mu \mathrm{m}(\mathbf{b}-\mathbf{d}), 70 \mu \mathrm{m}(\mathbf{e}), 5 \mu \mathrm{m}(\mathbf{f}), 10 \mu \mathrm{m}(\mathbf{g})$.

\section{Discussion}

The aim of the present study was to characterize the UGT72 family in poplar, a woody perennial plant, and to identify genes that could be involved in the glycosylation of monolignols. UGTs are known to show in vitro activities towards a large range of different substrates, and their substrate specificity cannot be predicted merely based on phylogenetic relationships [39]. As a first step in 
the characterization of the poplar UGT72 family, a phylogenetic tree of Arabidopsis and poplar UGT72 members was generated allowing the identification of 4 groups in poplar, of which 3 were common with Arabidopsis (Figure 2).

\subsection{Some Members of the Poplar UGT72 Family Are Involved in the Glycosylation of Monolignols}

Group 1 contains the Arabidopsis UGT72E1-E3 and UGT72C1 proteins, as well as the poplar UGT72AZ1 and UGT72AZ2 proteins. Recombinant Arabidopsis UGT72E1 uses coniferaldehyde and sinapaldehyde; UGT72E2 is able to glycosylate coniferyl and sinapyl alcohols, as well as coniferaldehyde, sinapaldehyde, and ferulic and sinapic acids; UGT72E3 displays activity towards coniferyl and sinapyl alcohols and sinapic acid; and UGT72C1 was found to glycosylate caffeic acid $[29,40,41]$. In brief, UGT72E2 is able to glycosylate monolignols and monolignol precursors, and UGT72E1 and UGT72E3 seem to be redundant to UGT72E2, at least partially. Here, in poplar, no activity towards any of the tested substrates was detected for UGT72AZ1. In contrast, UGT72AZ2 was found to glycosylate ferulic and sinapic acids but not (hydroxy)cinnamaldehydes nor (hydroxy)cinnamyl alcohols. In agreement with these results, it was previously observed that the recombinant Populus tomentosa PtUGT1, which is orthologous to UGT72AZ2, also showed no activity towards monolignols. Although belonging to the same phylogenetic group, these data indicate that the substrate specificity of poplar group 1 UGT72s is different from their Arabidopsis counterparts.

Metabolic analyses by HPLC were previously made on Arabidopsis overexpressing UGT72E1-E3 to measure the accumulation of glycosides of intermediates of the phenylpropanoid pathway. In the WT, only a low amount of coniferin was detected in rosette leaves [31]. In contrast, overexpression of UGT72E1, UGT72E2, and UGT72E3 led to a 6-fold, 85-fold, and 56-fold increase in coniferin content, respectively. An accumulation of syringin, ferulic acid 4-O-glucoside, and sinapic acid 4-O-glucoside was also detected in the transgenic plants overexpressing UGT72E2 and UGT72E3 [31,42]. Similarly, our results showed that coniferin and syringin accumulated in 35S-UGT72AZ1 lines and coniferin in 35S-UGT72AZ2 lines. The differences observed between UGT72 members in substrate specificity in vitro and in vivo may be explained by the availability of the appropriate substrates in planta $[2,43]$.

Group 2 includes the Arabidopsis UGT72D1 and UGT72D2P and the poplar UGT72A2. No activity towards cinnamic acids, cinnamaldehydes, nor cinnamyl alcohols has been found for UGT72D1 [28], and there is no data available for substrate specificity of UGT72D2P. The recombinant UGT72A2 protein did not use any of the tested phenylpropanoid substrates, and we did not observe increased accumulation of coniferin and syringin in the lines overexpressing UGT72A2. Further studies related to the identification of the substrates of these respective UGTs are needed to help to elucidate their function.

Group 3 comprises the poplar UGT72BB1, for which there is no corresponding Arabidopsis member. UGT72BB1 showed no activity towards the phenylpropanoids tested.

Finally, group 4 includes Arabidopsis UGT72B1-B3 and the poplar UGT72B36-B39. The recombinant UGT72B1 was reported to have glycosylating activity for $p$-coumaryl alcohol, coniferyl alcohol, dihydroconiferyl alcohol, $p$-coumaryl aldehyde, and coniferaldehyde. No activity was demonstrated for UGT72B2 towards the phenylpropanoids tested, and UGT72B3 showed activity on coniferaldehyde and sinapaldehyde [30]. Amongst the different members of the poplar UGT72 family, recombinant UGT72B37 glycosylated the highest number of substrates, including coniferyl and sinapyl alcohols, $p$-coumaraldehyde, coniferaldehyde, and sinapaldehyde, whereas UGT72B39 only used coniferyl alcohol. Based on the substrate specificities, UGT72B37 shows an enzymatic activity that covers that of Arabidopsis UGT72B1 and UGT72B3. However, in contrast to transgenic Arabidopsis lines overexpressing UGT72B1 that accumulate a higher amount of coniferin (up to 2-fold when compared to the WT [30]), no increase in the levels of monolignol glycosides were detected in leaves of transgenic poplar overexpressing UGT72B37 and UGT72B39. 


\subsection{UGT72s Are Expressed in the Vascular System}

The GUS expression driven by the poplar UGT72 promoters was observed in the vascular tissues for the investigated genes (Figure 6 and Figure S6). In Arabidopsis, UGT72 members are also expressed within vascular tissues. Concerning group 1, pUGT72E1 was found to be active in the root and $p U G T 72 E 2$, as well as pUGT72E3, were active in whole seedlings, in leaves, and in floral organs [31]. No data are available for $p U G T 72 C 1$. In poplar, $p U G T 72 A Z 1$ was active mainly in the phloem of the stem and $p U G T 72 A Z 2$ in the cambial zone of the root (Figure 6), and their corresponding proteins had a similar localization, as analyzed by immunolocalization (Figure 8).

For group 2, Arabidopsis pUGT72D1 was found to drive expression in phloem and in xylem [44]. In this study, poplar $p U G T 72 A 2$ was also active in phloem and xylem, and the corresponding protein accumulated correspondingly.

For group 4, in Arabidopsis, GUS staining of $p U G T 72 B 1:: G U S$ plants was found mainly in the xylem of the upper part of the stem and only in the xylem for older stem. For $p U G T 72 B 2$, GUS staining was mainly detected in veins of cotyledons and leaves and, for $p U G T 72 B 3$ in basal petiole, young leaf margins and slightly in the floral stem [30]. In poplar, the expression driven by the $p U G T 72 B 36$, pUGT72B37, and pUGT72B39 was found mainly in the primary xylem within the stem (Figure 6).

In silico analysis of the promoters revealed the presence of key cis-elements involved in vascular differentiation, such as SMRE, SNBE, AC-elements, and TERE [37,45-47]. The promoters of the UGT72B36-39 genes that were highly expressed in the xylem (Figure 5 and Figure S4) have several cis-elements related to xylem development, suggesting a possible role of these elements in their tissue-specific expression. In poplar, PtrMYB2, PtrMYB3, PtrMYB20, and PtrMYB21 are orthologous to Arabidopsis MYB46 and MYB83 [48,49]. They bind to the consensus SMRE domain $\mathrm{ACC}(\mathrm{A} / \mathrm{T}) \mathrm{A}(\mathrm{A} / \mathrm{C})(\mathrm{T} / \mathrm{C})$, which is found in the promoter of genes involved in cellulose, xylan, and lignin biosynthesis, as well as in $p U G T 72 B B 1, p U G T 72 B 36, p U G T 72 B 37$, and $p U G T 72 B 39$ (Figure 7). In Arabidopsis, MYB46 and MYB83 transcription factors also induce the expression of downstream transcription factors (MYB52, MYB63) and genes involved in secondary cell wall formation [50]. Some of these SMRE are identical to AC-elements, which are known to induce the expression of lignin biosynthesis genes [50]. The fact that the poplar UGT72B promoters contain elements recognized by these transcription factors, therefore, suggests that they are part of the transcriptional programme leading to secondary cell wall formation in xylem. Besides, single or several SNBE were also found in pUGT72s. These elements are targeted by NAC transcription factors, in which downstream-regulated genes partially overlap with MYB-induced genes involved in secondary cell wall formation [46,51], such as CesA4 and CesA8 [38,52]. NAC master transcription factors regulating secondary cell wall formation in Arabidopsis vessels and fibers include VND6, VND7, SND1, NST1, and NST2, which bind to the SNBE to enhance the expression of downstream transcription factors and biosynthesis genes [53]. SNBE are also found in genes expressed during xylem programmed cell death, such as Arabidopsis XYLEM CYSTEINE PEPTIDASE 1 and 2 [38] and poplar METACASPASE 13 and 14 [54]. Such genes are mainly induced by VND6 and VND7 [38,46]. Although the VND6/VND7 activation of genes carrying a TERE in the promoter region remains debated [38,46,53-55], this cis-element is enriched in genes involved in tracheary element differentiation, comprising cell death [38]. Our data therefore suggest that poplar UGT72s may also play a role during the late phase of xylem differentiation. We performed a similar analysis on promoters of Arabidopsis UGT72s (Table S5). Overall, they display the same cis-elements, suggesting that they may be regulated by the same set of MYB and NAC transcription factors. pUGT72D1 and pUGT72E1 each displayed six SNBE, while only one to three were detected in other Arabidopsis $p U G T 72 s$. Six cis-elements regulated by secondary cell wall-MYBs were present in pUGT72B3, possibly suggesting a different regulation.

\subsection{No Direct Impact of Poplar UGT72s Overexpression on Lignification Was Demonstrated}

Previously, two studies have shown that modifications in the expression level of particular members of the UGT72 family impact lignification. First, the ectopic expression of the P. tomentosa PtUGT1 
in tobacco induced an increase in approximately $60 \%$ in the Klason lignin amount in the stem [56]. As no enzymatic activity towards monolignols was found for the recombinant PtGT1, the effect of the overexpression of the corresponding gene on lignification was suggested to be probably indirect [56]. Second, the $u g t 72 b 1$ Arabidopsis mutant exhibits ectopic lignification [30]. The RNA-Seq analysis of the $u g t 72 b 1$ mutant showed modification of the expression of genes involved in the phenylpropanoid, flavonoid, and anthocyanin metabolisms. Particularly, genes involved in monolignol metabolism, possible monolignol transport ( $\mathrm{ABC}$ transporters), lignin polymerization, as well as regulation of secondary cell wall formation, were upregulated in this mutant, indicating feedback regulation upon disturbance of monolignol glucosylation on whole lignin synthesis, by a mechanism that awaits further discovery [30]. In the light of the large transcriptome reprogramming in the ugt72b1 mutant, the ectopic lignification phenotype is probably an indirect consequence of the mutation. This transcriptional shift is likely gene- and species-dependent, which may explain the absence of phenotype in lines overexpressing poplar UGT72 members (Figure S5). Further evidence for indirect effect of mutation on lignification is that both UGT72B1 overexpressor and ugt72b1 mutant lines produce increased amount of coniferin [30]. A role for UGT72s in defense response may be hypothesized. Actually, 35S-UGT72E2 Arabidopsis plants were more resistant to the fungus Verticillum longisporum than the WT, which was attributed to the accumulation of coniferin [57]. Based on an RNA-Seq analysis, UGT72E1 and UGT72E2, together with UGT71C1, were found to be the most upregulated UGT genes in Arabidopsis in response to infection by the fungus Plasmodiophora brassicae [58], suggesting that the corresponding proteins might be directly or indirectly involved in pathogen resistance. Further analysis of responses to pathogen infection of transgenic poplar overexpressing UGT72AZ1/AZ2 and UGT72B37/B39 may help to unravel the role of glycosylated monolignols in disease resistance or in developmental lignification.

\subsection{Differences in Subcellular Localization of Poplar UGT72s May Indicate Divergent Functions among the Different Members of the Same Family}

At the subcellular level, poplar UGT72s from group 1 (UGT72AZ1-2) and group 4 (UGT72B36-37 and 39) were localized in the nucleus and associated with the ER (Figure 9). Accordingly, several nuclear localization signals (NLSs) were detected in all UGT72s from poplar (cNLS mapper [59]). This analysis indicated a double localization, both in the nucleus and in the cytoplasm (Table S6). Several enzymes of the monolignol biosynthesis pathway (Figure 1), such as $\mathrm{C} 4 \mathrm{H}, \mathrm{C} 3 \mathrm{H}, \mathrm{F} 5 \mathrm{H}$, and $\mathrm{HCT}$, are, at least partly, associated with the ER [60,61]. UGT84A23-GFP, involved in gallic acid glycosylation, is also possibly targeted to the ER [62]. Monolignol homeostasis may therefore rely on the activity of UGTs which colocalize with monolignol enzymes to directly glycosylate some of the intermediate products of this metabolic pathway to circumvent potential toxicity. Such a mechanism has been suggested for a vanillin-specific UGT in order to maintain a content at sub-toxic levels in the cell [63]. Assembly and disassembly of a given metabolon, for instance, an UGT coupled to a P450 protein, would provide additional flexibility in specialized metabolite biosynthesis. As an illustration, soluble UGT85B1 was found to be recruited to the ER, in close vicinity to enzymes of the same biosynthetic pathway, to favor dhurrin biosynthesis in response to insect attack [64]. Besides, UGT72E1 was previously identified by yeast two-hybrid as an interacting partner of SIS8, a putative mitogen-activated protein kinase kinase kinase, and these proteins were both localize in the nucleus when transiently expressed in tobacco leaf cells [32].

In contrast, UGT72A2 (group 2) was found to be associated with chloroplastic bodies, as well as possibly localized within chloroplasts, suggesting a different function than the other UGT72s from poplar. More precisely, UGT72A2-GFP was localized in bodies associated with the chloroplast following tobacco transient transformation with Agrobacterium, while, in stable transgenic lines carrying the same construct, the signal was also detected inside chloroplasts. These bodies are usually observed when large quantities of proteins are present, either due to high expression driven by the CaMV35S promoter or when high quantities of plasmids are used for the transfection $[65,66]$. We may speculate that, in stable transgenic poplars, the biosynthesis of proteins is more regulated; therefore, UGT72A2 is 
restricted to its native compartment, i.e., chloroplast. Finally, TargetP analysis [67] in poplar UGT72s for the presence of $\mathrm{N}$-terminal presequences targeting the protein to a specific compartment did not reveal any preferential localization.

\section{Materials and Methods}

\subsection{Plant Material and Growth Conditions}

Both WT and transgenic poplars (Populus tremula $\times$ P. alba, clone INRA 717-1B4) were grown aseptically or in the greenhouse, as described previously [68].

\subsection{Cloning of UGT72s Coding and Promoter Sequences from P. Tremula $\times$ P. Alba}

Arabidopsis UGT72s protein sequences were used to screen the $P$. trichocarpa genome sequence (https://phytozome.jgi.doe.gov/pz/portal.html). Ten polypeptides sharing 45\% identity with Arabidopsis UGT were identified. P. trichocarpa coding sequences were then used to identify corresponding P. tremula $\times$ P. alba coding sequences using BLASTN in the P. tremula $\times$ P. alba clone INRA 717-1B4 genome sequence (https://urgi.versailles.inra.fr/Species/Forest-trees/Populus/CloneINRA-717-1B4). P. tremula $\times$ P. alba gene specific primers were designed using PerlPrimer (v1.1.21) (Table S1) to amplify either corresponding cDNA or approximately $2 \mathrm{~kb}(1.6-2.1 \mathrm{~kb}$ depending on the gene) DNA region upstream of the start codon. The different UGT72s' coding and promoter sequences were then cloned in pCR4 TOPO or pCR Blunt II (Life Technologies, Carlsbad, CA, USA) and sequenced. Cis-elements related to secondary cell wall formation were retrieved from the promoter $1500 \mathrm{bp}$ upstream of the start codon with QIAGEN CLC Genomics Workbench (v20) and visualized online with IBS [69].

\subsection{Phylogenetic Analysis}

Full-length protein sequence alignment (MUSCLE) and generation of the phylogenetic tree using the approximate likelihood-ratio test (SH-like) in PhyML was performed online (phylogeny.fr, [70,71]).

\subsection{Expression Profile Analysis by RT-qPCR and Histochemical GUS Staining}

Total RNA was extracted from stems and roots of 4-month-old P. tremula $\times P$. alba $(n=3$, one tree per biological replicate) grown in the greenhouse using the Agilent Plant RNA isolation mini kit (Agilent, Santa Clara, CA, USA). Within the stem, three samples were harvested. The first one corresponded to the 2 first $\mathrm{cm}$ of the stem, including the apex and leaf primordia, corresponding to primary VT. For the second and third samples, a portion of the stem was removed at $50 \mathrm{~cm}$ below the apex (corresponding to secondary VT) and separated into bark (phloem) and wood (xylem). For the roots, primary VT corresponded to young root with primary structure, and secondary VT corresponded to roots with a diameter of $1 \mathrm{~cm}$. The reverse transcription was carried out with GoScript Reverse Transcription Mix, Oligo(dT) kit (Promega, Madison, WI, USA). The qPCR was carried out with Luna Universal qPCR Master Mix and the LightCycler 480 system (Roche, Bâle, Switzerland) in technical triplicate. Amplification specificity was checked at the end of each qPCR run with a melt curve. The obtained values were normalized with two reference genes (CYC063 and CDC2 [72]). The gene relative expression was calculated using specific primers efficiency. Primers sequences are shown in Table S1.

Histochemical GUS staining was performed as described by Hemerly et al. [73] with modifications. After fixation in $90 \%$ acetone $\left(1 \mathrm{~h}\right.$ at $\left.-20{ }^{\circ} \mathrm{C}\right)$, samples were rinsed with PBS and incubated with GUS reaction buffer (50 mM PBS, $0.1 \%$ triton, $0.1 \mathrm{mM}$ potassium ferricyanide, $0.1 \mathrm{mM}$ potassium ferrocyanide, and $0.3 \mathrm{mg} / \mathrm{mL}$ 5-bromo-4-chloro-3-indolyl $\beta$-D-glucuronic acid (X-Gluc)). After GUS staining overnight, samples were decolored in 70\% ethanol, and cross sections $(60-100 \mu \mathrm{m}$ thick) were made with an HM $650 \mathrm{~V}$ vibratome (Thermo Scientific, Waltham, MA, États-Unis, USA, http://www.thermoscientific.com) and observed under a light microscope. 


\subsection{Antibody Production, Immunoblotting and Immunolocalization}

Polyclonal antibodies raised against specific peptides of UGT72AZ1 (TDNSLSKSQLLKQSPC), UGT72AZ2 (CKSVRFEDTLPAYLDR), and UGT72A2 (LTRPLKPLDSRSGEL) were produced and quality-checked externally (Eurogentec, Liège, Belgium). The cross-reactivity of the purified antibodies was tested by immunoblot with the recombinant proteins UGT72AZ1, AZ2, and A2 produced in E. coli BL21(DE3) strain in the vector pBAD-DEST49 (Invitrogen, Carlsbad, CA, États-Unis, USA) (Figure S7). The expression of the vector was induced with $0.02 \%$ arabinose $\left(2 \mathrm{~h}\right.$ at $\left.37^{\circ} \mathrm{C}\right)$. Proteins from induced culture were extracted from the crude extract and separated using an SDS-PAGE using a $12.5 \%$ acrylamide resolving gel with a Criterion Cell device (Bio-rad, Hercules, CA, USA). After the migration, proteins were transferred to PVDF Hybond-P membrane (Amersham Biosciences). The membrane was blocked with Odyssey blocking buffer (LI-COR Biosciences, Lincoln, NE, USA), then incubated overnight at $4{ }^{\circ} \mathrm{C}$ with primary antibody (at a dilution of $1 / 200$ for anti-UGT72AZ1, 1/1000 for anti-UGT72AZ2 and 1/200 for anti-UGT72A2) in PBS buffer solution with $0.2 \%$ of Tween 20 (Sigma-Aldrich, Saint-Louis, MI, USA). Subsequently, it was washed thrice with TBST (Tris 1M $\mathrm{pH} 8, \mathrm{NaCl} 5 \mathrm{M}$ and $0.1 \%$ of Tween 20 ) and incubated with secondary antibodies (goat anti-rabbit immunoglobulin-G) diluted 1/10,000 in PBS buffer solution with $0.2 \%$ of Tween 20 at $37^{\circ} \mathrm{C}$ during $1 \mathrm{~h}$ followed by 3 washes in TBST. Signals were revealed with Odyssey Fc Imaging System (LI-COR Biosciences). The image processing was realized with Image Studio Lite (LI-COR biosciences).

For immunolocalization, stem pieces were fixed with a solution of $4 \%$ formaldehyde and $0.1 \%$ Triton in $50 \mathrm{mM}$ PIPES overnight at $4{ }^{\circ} \mathrm{C}$ with agitation after a vacuum for $30 \mathrm{~min}$. After fixation, stem sections $(100 \mu \mathrm{m})$ obtained using an HM $650 \mathrm{~V}$ vibratome were incubated with primary antibodies (diluted at 1/100 for anti-UGT72AZ1, 1/200 for anti-UGT72AZ2, and 1/100 for anti-UGT72A2 in 0.75x Odyssey blocking buffer $+0.2 \%$ DMSO) overnight at $4{ }^{\circ} \mathrm{C}$. Sections were washed thrice with $50 \mathrm{mM}$ PIPES buffer, incubated for $1 \mathrm{~h} 30 \mathrm{~min}$ at $37^{\circ} \mathrm{C}$ with anti-rabbit antibodies conjugated to AlexaFluor 488 (1/500; Life Technologies) and washed thrice with $50 \mathrm{mM}$ PIPES buffer before observation using a Zeiss confocal microscope LSM710. Secondary antibody was detected with the following parameters: excitation at $488 \mathrm{~nm}$, emission recorded between 495 and $573 \mathrm{~nm}$. Lignin autofluorescence was detected with the following parameters: excitation at $405 \mathrm{~nm}$, emission recorded between 410 and $507 \mathrm{~nm}$. All acquisition settings were similar for sections incubated with the different antibodies, and no change was made to image display post-acquisition.

\subsection{Subcellular Localization of the poplar UGT72s Fused with GFP}

Poplar UGT72s were cloned in the vector pK7FWG2 [74] by Gateway technology. 35S::AtWAK2-mCherry construct was used as marker for ER [75]. Each construct was used to transform A. tumefaciens (strain C58RifPMP90) by electroporation. After separated preculture in LB medium with $10 \mathrm{mM}$ MES buffer and $20 \mu \mathrm{M}$ acetosyringone (supplemented with the selection antibiotic), bacterial suspensions were mixed, and agroinfiltration were performed in tobacco leaves. After 2 to 4 days, leaves were analyzed with a LSM710 confocal microscope. The wavelength of excitation for GFP is $488 \mathrm{~nm}$, and the emission was recorded between 495 and $543 \mathrm{~nm}$. The wavelength of excitation for mCherry is $543 \mathrm{~nm}$, and the emission was recorded between 560 and $630 \mathrm{~nm}$. The analysis was not performed for UGT72BB1 and UGT72B38.

\subsection{Enzymatic Assay on Recombinant Proteins Produced in E. Coli}

Recombinant protein production was performed in E. coli BL21(DE3)pLysS genotype using UGT72s cloned into pGEX-4T-1 (GE Healthcare, Chicago, IL, USA). Following transformation, a preculture grown at $37^{\circ} \mathrm{C}$ was used to inoculate $500 \mathrm{~mL}$ of LB supplemented with ampicillin $(100 \mu \mathrm{g} / \mathrm{mL})$ and chloramphenicol $(34 \mu \mathrm{g} / \mathrm{mL}$ ). Induction with IPTG (final concentration of $1 \mathrm{mM}$ ) was performed at an $\mathrm{OD}_{600}$ of $0.5-0.6$, followed by overnight incubation at $18{ }^{\circ} \mathrm{C}$ under agitation $(200 \mathrm{rpm})$. Cells were then pelleted, washed, and stored at $-80^{\circ} \mathrm{C}$ until protein purification. The pellet was then resuspended 
in $10 \mathrm{~mL}$ GST-Wash/Bind buffer supplemented with PMSF (final concentration $1 \mathrm{mM}$ ) and lysozyme and ultrasonicated. The resulting bacterial lysate was centrifuged $\left(30 \mathrm{~min}, 13,500 \mathrm{rpm}\right.$ at $\left.4{ }^{\circ} \mathrm{C}\right)$, and the supernatant was applied to a column with GST•Bind ${ }^{\mathrm{TM}}$ resin (Novagen). GST-tagged proteins were allowed to bind to the resin overnight at $4{ }^{\circ} \mathrm{C}$ under soft agitation. After three washings, proteins were eluted in 5 consecutive fractions ( $300 \mu \mathrm{L}$ each). The purity of these 5 fractions was investigated through SDS-PAGE, before pooling the pure fractions for quantification with the Roti-Nanoquant assay (Carl Roth).

The enzymatic assay was performed with $5 \mu \mathrm{g}$ of purified protein and the following final concentrations: $100 \mathrm{mM}$ Tris $\mathrm{HCl}$ pH7.5, $1 \mathrm{mM}$ UDP-glucose, $600 \mu \mathrm{M}$ substrate (coniferaldehyde, coniferyl alcohol, $p$-coumaraldehyde, sinapaldehyde, sinapyl alcohol, trans-cinnamic acid, $p$-coumaric acid, caffeic acid, ferulic acid, sinapic acid, and salicylic acid) in a total volume of $100 \mu \mathrm{L}$. Each reaction was performed in triplicate and incubated overnight at $30^{\circ} \mathrm{C}$. The reaction was stopped by heating at $75{ }^{\circ} \mathrm{C}$ for $10 \mathrm{~min}$, centrifuged, and analyzed with a LC-UV-MS/MS system using the protocol described in Reference [76]. Identity of the glycosylated product was established by examination of the MS/MS spectra.

\subsection{Generation of Transgenic Poplars Upregulating UGT72s, Expressing Promoter-GUS or UGT72A2-GFP Constructs}

For the overexpression, UGT72s were cloned in PK7WG2D [74] and promoter-GUS constructs were obtained by inserting the different promoters into pKGWFS7 [74] by Gateway technology. The UGT72A2-GFP construct is the same as that used for agroinfiltration. The recombined plasmids

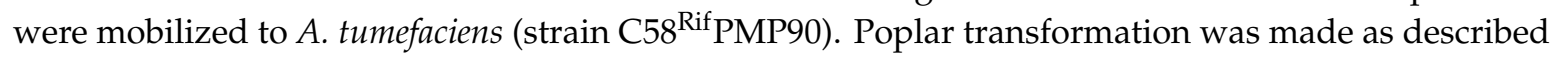
in [77]. A minimum of 20 independent transgenic lines per construct were selected based on kanamycin resistance.

\subsection{Lignin Content}

Lignin content was determined in cell wall residue [78] made from wood and bark of stem and root of 4-month-old transgenic poplars and WT (at least in biological triplicate) using acetyl bromide digestion, as described previously [79].

\subsection{Metabolite Extraction, HPLC-UV-MS and LC-MS Analyses}

Plant material was ground in liquid nitrogen for each line (at least in biological triplicate). Metabolites were extracted on $200 \mathrm{mg}$ (fresh weight) with $1 \mathrm{~mL}$ methanol $80 \%$ on ice over $4 \mathrm{~h}$ with several vortexing steps. After centrifugation, the extract was filtered on a $0.22 \mu \mathrm{m}$ membrane, aliquoted, and $20 \mu \mathrm{L}$ were injected in a HPLC-UV-MS system (Agilent 1260 Infinity II coupled to Advion Expression CMS L). Metabolites were separated using a water ( $0.1 \%$ formic acid)/acetonitrile gradient (10 to $30 \% \mathrm{ACN}$ in $20 \mathrm{~min}$ ) at $1 \mathrm{~mL} /$ minute on a C18 column (InfinityLab Poroshell 120 EC-C18, $100 \times 4.6 \mathrm{~mm}$ i.d., $4 \mu \mathrm{m})$. Identification of coniferin and syringin was based on their retention time, absorption at $264 \mathrm{~nm}$ and mass spectra by comparison with authentic standards. Differences in phenolic abundance between WT and overexpressing lines were assessed by comparing UV profiles at $264 \mathrm{~nm}$.

Accumulation of coniferin and syringin in UGT72AZ1 and UGT72AZ2 overexpressing lines was measured through LC-MS analysis on an ACQUITY UPLC I-Class system (Waters Corporation, Milford, MA, USA), coupled to a Synapt QTOF hybrid mass spectrometer (Waters Corporation). Chromatographic separation was carried out on an ACQUITY UPLC BEH C18 $(150 \times 2.1 \mathrm{~mm}, 1.7 \mu \mathrm{m})$ column from Waters, temperature was maintained at $40{ }^{\circ} \mathrm{C}$. A 30 min gradient of two buffers was used: buffer $\mathrm{A}$ (99/1/0.1 $\mathrm{H}_{2} \mathrm{O} / \mathrm{ACN} /$ formic acid $\left.\mathrm{pH} 3\right)$, buffer $\mathrm{B}$ (99/1/0.1 ACN/ $\mathrm{H}_{2} \mathrm{O} /$ formic acid $\mathrm{pH} 3$ ); $95 \%$ A for $0.1 \mathrm{~min}$ decreased to $50 \% \mathrm{~A}$ in $30 \mathrm{~min}$. The flow rate was set to $0.35 \mathrm{~mL} / \mathrm{minute}$, and the injection volume was $15 \mu \mathrm{L}$. The MS LockSpray ion source was operated in negative electrospray ionization (ESI) mode under the following specific conditions: capillary voltage: $2.5 \mathrm{kV}$; source temperature; $120^{\circ} \mathrm{C}$; 
desolvation gas temperature; $400{ }^{\circ} \mathrm{C}$; desolvation gas flow; $550 \mathrm{~L} / \mathrm{h}$. Mass range was set from $100-1000$ Da. Nitrogen (>99.5\%) was employed as desolvation and cone gas. Leucin-enkephalin $(250 \mathrm{pg} / \mu \mathrm{L}$ solubilized in water/ACN $1: 1(v / v)$, with $0.1 \%$ formic acid) was used for the lock mass calibration. Centroided data was recorded through Masslynx software (Waters). Chromatogram processing and data normalization was performed with Progenesis QI software v2.1 (Waters). Database maintenance for compound identification was done with JChem for Excel add-in (Chemaxon, Budapest, Hungary).

\subsection{Accession Uumbers}

Gene sequence data from this article are deposited in GenBank under the accession numbers MT181026 (UGT72A2), MT181027 (UGT72AZ1), MT181028 (UGT72AZ2), MT181029 (UGT72B36), MT181030 (UGT72B37), MT181031 (UGT72B38), MT181032 (UGT72B39), MT181033 (UGT72BB1), MT193261 ( $p$ UGT72A2), MT193262 (pUGT72AZ1), MT193263 (pUGT72AZ2), MT193264 (pUGT72B36), MT193265 (pUGT72B37), MT193266 (pUGT72B38), MT193267 ( $p$ UGT72B39), and MT193268 ( $p U G T 72 B B 1)$.

The accession numbers of the Arabidopsis UGT72s are At3G50740 (UGT72E1), At5G26310 (UGT72E2), At5G66690 (UGT72E3), At4G01070 (UGT72B1), At1g01390 (UGT72B2), At1g01420 (UGT72B3), At4G36770 (UGT72C1), At2G18570 (UGT72D1), and At2G18560 (UGT72D2P). The P. trichocarpa accession numbers corresponding to the cloned UGT72s from P. tremula $\times$ P. alba are Potri.007G030300 (UGT72AZ1), Potri.007G030400 (UGT72AZ2), Potri.007G030500 (UGT72A2), Potri.014G041800 (UGT72BB1), Potri.014G096000 (UGT72B36), Potri.014G096100 (UGT72B37), Potri.003G138200 (UGT72B38), and Potri.002G168600 (UGT72B39).

\section{Conclusions}

The survey of the UGT72 family in poplar allowed the identification of members that are involved in the glycosylation of monolignols and that are clustered with Arabidopsis UGT72Es (group 1) and UGT72Bs (group 4). In addition, poplar UGT72s, like their Arabidopsis counterparts, are expressed within vascular tissues. No direct impact of the overexpression of poplar UGT72s on lignification was evidenced. Finally, the observed differences in subcellular localization suggest divergent functions of members of the poplar UGT72 family. Further studies on poplar mutants and identification of substrates are required to unravel the role of UGT72s, particularly in the lignification process or in defense towards pathogens in poplar.

Supplementary Materials: Supplementary materials can be found at http://www.mdpi.com/1422-0067/21/14/ 5018/s1.

Author Contributions: Conceptualization, N.S., F.B.-C., M.E.J., M.B. (Marc Behr), and M.B. (Marie Baucher); formal analysis, N.S., N.M.A., H.A.H., F.B.-C., G.G., and M.B. (Marc Behr); funding acquisition, M.B. (Marie Baucher); investigation, N.S., N.M.A., H.A.H., F.B.-C., A.M., G.G., and M.B. (Marc Behr); project administration, M.B. (Marie Baucher); resources, W.B., P.D., S.H., G.N. and W.S.; supervision, F.B.-C., M.E.J., M.B. (Marc Behr), and M.B. (Marie Baucher); visualization, N.S., N.M.A., H.A.H., F.B.-C., and M.B. (Marc Behr); writing-original draft, M.E.J., M.B. (Marc Behr), and M.B. (Marie Baucher); writing-review and editing, F.B.-C., W.B., P.D., S.H., G.N., T.H., W.S., M.E.J., M.B. (Marc Behr), and M.B. (Marie Baucher). All authors have read and agreed to the published version of the manuscript.

Funding: M. Behr is supported by Belgian Fonds de la Recherche Scientifique (FRS-FNRS) research project T.0068.18, and M. Baucher is a Senior Research Associate of the FRS-FNRS. N.S. was the recipient of pre-doctoral grants from the "Fonds pour la Formation à la Recherche dans l'Industrie et l'Agriculture (FRIA), N.M.A. and H.A.H. from the Académie de Recherche et d'Enseignement Supérieur, Belgium (ARES), respectively.

Acknowledgments: The authors would like to acknowledge Rebecca Van Acker, Amana Metuor Dabire, Mouhamadou Mounkaila Boureima, Razacwendé Fanta Ouédraogo and Oriane Morel for valuable assistance in lignin analysis and molecular biology lab work and Claudio Palmieri (UMONS) for excellent technical assistance with HPLC-UV-MS analysis. M. Behr is grateful for the support of researchers from Wilfried Schwab's lab for enzymatic activities. Confocal microscopy was performed at the CMMI. The CMMI is supported by the European Regional Development Fund and the Walloon Region. This article is published with the assistance of the Belgian University Foundation. 
Conflicts of Interest: The authors declare no conflict of interest. The funders had no role in the design of the study; in the collection, analyses, or interpretation of data; in the writing of the manuscript, or in the decision to publish the results.

\section{References}

1. Lairson, L.L.; Henrissat, B.; Davies, G.J.; Withers, S.G. Glycosyltransferases: Structures, Functions, and Mechanisms. Annu. Rev. Biochem. 2008, 77, 521-555. [CrossRef] [PubMed]

2. Bowles, D.; Lim, E.-K.; Poppenberger, B.; Vaistij, F.E. Glycosyltransferases of lipophilic small molecules. Annu. Rev. Plant Biol. 2006, 57, 567-597. [CrossRef] [PubMed]

3. Le Roy, J.; Huss, B.; Creach, A.; Hawkins, S.; Neutelings, G. Glycosylation is a major regulator of phenylpropanoid availability and biological activity in plants. Front. Plant Sci. 2016, 7, 735. [CrossRef]

4. Kumar, V.; Hainaut, M.; Delhomme, N.; Mannapperuma, C.; Immerzeel, P.; Street, N.R.; Henrissat, B.; Mellerowicz, E.J. Poplar carbohydrate-active enzymes: Whole-genome annotation and functional analyses based on RNA expression data. Plant J. 2019, 99, 589-609. [CrossRef] [PubMed]

5. Yonekura-Sakakibara, K.; Hanada, K. An evolutionary view of functional diversity in family 1 glycosyltransferases. Plant J. 2011, 66, 182-193. [CrossRef]

6. Hughes, J.; Hughes, M.A. Multiple secondary plant product UDP-glucose glucosyltransferase genes expressed in cassava (Manihot esculenta Crantz) cotyledons. DNA Seq. 1994, 5, 41-49. [CrossRef] [PubMed]

7. Osmani, S.A.; Bak, S.; Møller, B.L. Substrate specificity of plant UDP-dependent glycosyltransferases predicted from crystal structures and homology modeling. Phytochemistry 2009, 70, 325-347. [CrossRef] [PubMed]

8. Tiwari, P.; Sangwan, R.S.; Sangwan, N.S. Plant secondary metabolism linked glycosyltransferases: An update on expanding knowledge and scopes. Biotechnol. Adv. 2016, 34, 714-739. [CrossRef] [PubMed]

9. Boerjan, W.; Ralph, J.; Baucher, M. Lignin biosynthesis. Annu. Rev. Plant Biol. 2003, 54, 519-546. [CrossRef]

10. Tsuyama, T.; Takabe, K. Distribution of lignin and lignin precursors in differentiating xylem of Japanese cypress and poplar. J. Wood Sci. 2014, 60, 353-361. [CrossRef]

11. Freudenberg, K.; Harkin, J.M. The glucosides of cambial sap of spruce. Phytochemistry 1963, 2, $189-193$. [CrossRef]

12. Rolando, C.; Daubresse, N.; Pollet, B.; Jouanin, L.; Lapierre, C. Lignification in poplar plantlets fed with deuterium-labelled lignin precursors. C. R. Biol. 2004, 327, 799-807. [CrossRef] [PubMed]

13. Terazawa, M.; Okuyama, H.; Miyake, M. Isolation of coniferin and syringin from the cambial tissue and inner-bark of some angiospermous woods. J. Jpn. Wood Res. Soc. 1984, 30, 409-412.

14. Fukushima, K.; Taguchi, S.; Matsui, N.; Yasuda, S. Heterogeneous distribution of monolignol glucosides in the stems of Magnolia kobus. Mokuzai Gakkaishi 1996, 42, 1029-1031.

15. Aoki, D.; Okumura, W.; Akita, T.; Matsushita, Y.; Yoshida, M.; Sano, Y.; Fukushima, K. Microscopic distribution of syringin in freeze-fixed Syringa vulgaris stems. Plant Direct 2019, 3, e00155. [CrossRef]

16. Aoki, D.; Nomura, K.; Hashiura, M.; Imamura, Y.; Miyata, S.; Terashima, N.; Matsushita, Y.; Nishimura, H.; Watanabe, T.; Katahira, M.; et al. Evaluation of ring-5 structures of guaiacyl lignin in Ginkgo biloba L. using solid- and liquid-state 13C NMR difference spectroscopy. Holzforschung 2019, 73, 1083-1092. [CrossRef]

17. Aoki, D.; Matsushita, Y.; Fukushima, K. Cryo-TOF-SIMS visualization of water-soluble compounds in plants. In ACS Symposium Series; American Chemical Society: Washington, DC, USA, 2018; Volume 1286, pp. 137-150. ISBN 9780841232969.

18. Aoki, D.; Hanaya, Y.; Akita, T.; Matsushita, Y.; Yoshida, M.; Kuroda, K.; Yagami, S.; Takama, R.; Fukushima, K. Distribution of coniferin in freeze-fixed stem of Ginkgo biloba L. by cryo-TOF-SIMS/SEM. Sci. Rep. 2016, 6, 31525. [CrossRef] [PubMed]

19. Terashima, N. Non-destructive approaches to identify the ultrastructure of lignified ginkgo cell walls. Int. J. Dev. Biol. 2007, 1, 170-177.

20. Tsuji, Y.; Fukushima, K. Behavior of monolignol glucosides in angiosperms. J. Agric. Food Chem. 2004, 52, 7651-7659. [CrossRef] [PubMed]

21. Väisänen, E.E.; Smeds, A.I.; Fagerstedt, K.V.; Teeri, T.H.; Willför, S.M.; Kärkönen, A. Coniferyl alcohol hinders the growth of tobacco BY-2 cells and Nicotiana benthamiana seedlings. Planta 2015, 242, 747-760. [CrossRef] 
22. Liu, C.J. Deciphering the enigma of lignification: Precursor transport, oxidation, and the topochemistry of lignin assembly. Mol. Plant 2012, 5, 304-317. [CrossRef] [PubMed]

23. Wang, Y.; Chantreau, M.; Sibout, R.; Hawkins, S. Plant cell wall lignification and monolignol metabolism. Front. Plant Sci. 2013, 4, 220. [CrossRef] [PubMed]

24. Dima, O.; Morreel, K.; Vanholme, B.; Kim, H.; Ralph, J.; Boerjan, W. Small glycosylated lignin oligomers are stored in Arabidopsis leaf vacuoles. Plant Cell 2015, 27, 695-710. [CrossRef] [PubMed]

25. Miao, Y.C.; Liu, C.J. ATP-binding cassette-like transporters are involved in the transport of lignin precursors across plasma and vacuolar membranes. Proc. Natl. Acad. Sci. USA 2010, 107, 22728-22733. [CrossRef]

26. Alejandro, S.; Lee, Y.; Tohge, T.; Sudre, D.; Osorio, S.; Park, J.; Bovet, L.; Lee, Y.; Geldner, N.; Fernie, A.R.; et al. AtABCG29 is a monolignol transporter involved in lignin biosynthesis. Curr. Biol. 2012, 22, 1207-1212. [CrossRef]

27. Vermaas, J.V.; Dixon, R.A.; Chen, F.; Mansfield, S.D.; Boerjan, W.; Ralph, J.; Crowley, M.F.; Beckham, G.T. Passive membrane transport of lignin-related compounds. Proc. Natl. Acad. Sci. USA 2019, 116, 23117-23123. [CrossRef]

28. Lim, E.K.; Li, Y.; Parr, A.; Jackson, R.; Ashford, D.A.; Bowles, D.J. Identification of glucosyltransferase genes involved in sinapate metabolism and lignin synthesis in Arabidopsis. J. Biol. Chem. 2001, 276, 4344-4349. [CrossRef]

29. Lim, E.-K.; Jackson, R.G.; Bowles, D.J. Identification and characterisation of Arabidopsis glycosyltransferases capable of glucosylating coniferyl aldehyde and sinapyl aldehyde. FEBS Lett. 2005, 579, 2802-2806. [CrossRef]

30. Lin, J.S.; Huang, X.X.; Li, Q.; Cao, Y.; Bao, Y.; Meng, X.F.; Li, Y.J.; Fu, C.; Hou, B.K. UDP-glycosyltransferase 72B1 catalyzes the glucose conjugation of monolignols and is essential for the normal cell wall lignification in Arabidopsis thaliana. Plant J. 2016, 88, 26-42. [CrossRef] [PubMed]

31. Lanot, A.; Hodge, D.; Lim, E.K.; Vaistij, F.E.; Bowles, D.J. Redirection of flux through the phenylpropanoid pathway by increased glucosylation of soluble intermediates. Planta 2008, 228, 609-616. [CrossRef]

32. Huang, Y.; Li, C.Y.; Qi, Y.; Park, S.; Gibson, S.I. SIS8, a putative mitogen-activated protein kinase kinase kinase, regulates sugar-resistant seedling development in Arabidopsis. Plant J. 2014, 77, 577-588. [CrossRef] [PubMed]

33. Lefevere, H.; Bauters, L.; Gheysen, G. Salicylic acid biosynthesis in plants. Front. Plant Sci. 2020, 11, 338. [CrossRef]

34. Shi, R.; Wang, J.P.; Lin, Y.-C.; Li, Q.; Sun, Y.-H.; Chen, H.; Sederoff, R.R.; Chiang, V.L. Tissue and cell-type co-expression networks of transcription factors and wood component genes in Populus trichocarpa. Planta 2017, 245, 927-938. [CrossRef] [PubMed]

35. Sundell, D.; Street, N.R.; Kumar, M.; Mellerowicz, E.J.; Kucukoglu, M.; Johnsson, C.; Kumar, V.; Mannapperuma, C.; Delhomme, N.; Nilsson, O.; et al. Aspwood: High-spatial-resolution transcriptome profiles reveal uncharacterized modularity of wood formation in Populus tremula. Plant Cell 2017, 29, 1585-1604. [CrossRef]

36. Zhong, R.; Ye, Z.H. Secondary cell walls: Biosynthesis, patterned deposition and transcriptional regulation. Plant Cell Physiol. 2015, 56, 195-214. [CrossRef]

37. Pyo, H.; Demura, T.; Fukuda, H. TERE; a novel cis-element responsible for a coordinated expression of genes related to programmed cell death and secondary wall formation during differentiation of tracheary elements. Plant J. 2007, 51, 955-965. [CrossRef]

38. Yamaguchi, M.; Mitsuda, N.; Ohtani, M.; Ohme-Takagi, M.; Kato, K.; Demura, T. VASCULAR-RELATED NAC-DOMAIN 7 directly regulates the expression of a broad range of genes for xylem vessel formation. Plant J. 2011, 66, 579-590. [CrossRef]

39. Yang, M.; Fehl, C.; Lees, K.V.; Lim, E.K.; Offen, W.A.; Davies, G.J.; Bowles, D.J.; Davidson, M.G.; Roberts, S.J.; Davis, B.G. Functional and informatics analysis enables glycosyltransferase activity prediction. Nat. Chem. Biol. 2018, 14, 1109-1117. [CrossRef]

40. Lim, E.-K.; Baldauf, S.; Li, Y.; Elias, L.; Worrall, D.; Spencer, S.P.; Jackson, R.G.; Taguchi, G.; Ross, J.; Bowles, D.J. Evolution of substrate recognition across a multigene family of glycosyltransferases in Arabidopsis. Glycobiology 2003, 13, 139-145. [CrossRef] 
41. Han, D.Y.; Lee, H.R.; Kim, B.G.; Ahn, J.H. Biosynthesis of ferulic acid 4-O-glucoside and feruloyl glucoside using Escherichia coli harboring regioselective glucosyltransferases. Appl. Biol. Chem. 2016, 59, 481-484. [CrossRef]

42. Lanot, A.; Hodge, D.; Jackson, R.G.; George, G.L.; Elias, L.; Lim, E.-K.; Vaistij, F.E.; Bowles, D.J. The glucosyltransferase UGT72E2 is responsible for monolignol 4-O-glucoside production in Arabidopsis thaliana. Plant J. 2006, 48, 286-295. [CrossRef]

43. Achnine, L.; Huhman, D.V.; Farag, M.A.; Sumner, L.W.; Blount, J.W.; Dixon, R.A. Genomics-based selection and functional characterization of triterpene glycosyltransferases from the model legume Medicago truncatula. Plant J. 2005, 41, 875-887. [CrossRef]

44. Chapelle, A. Caractérisation de gènes de $\beta$-glucosidase et d'UDP-glycosyltransférase potentiellement impliqués dans la lignification chez Arabidopsis thaliana. Ph.D. Thesis, Université Paris-Sud, Orsay, France, 2009.

45. McCarthy, R.L.; Zhong, R.; Ye, Z.H. Secondary wall NAC binding element (SNBE), a key Cis-acting element required for target gene activation by secondary wall NAC master switches. Plant Signal. Behav. 2011, 6, 1282-1285. [CrossRef]

46. Ohashi-Ito, K.; Oda, Y.; Fukuda, H. Arabidopsis VASCULAR-RELATED NAC-DOMAIN6 directly regulates the genes that govern programmed cell death and secondary wall formation during xylem differentiation. Plant Cell 2010, 22, 3461-3473. [CrossRef]

47. Kim, W.C.; Ko, J.H.; Han, K.H. Identification of a cis-acting regulatory motif recognized by MYB46, a master transcriptional regulator of secondary wall biosynthesis. Plant Mol. Biol. 2012, 78, 489-501. [CrossRef]

48. Zhong, R.; McCarthy, R.L.; Haghighat, M.; Ye, Z.-H. The poplar MYB master switches bind to the SMRE site and activate the secondary wall biosynthetic program during wood formation. PLOS ONE 2013, 8, e69219. [CrossRef] [PubMed]

49. McCarthy, R.L.; Zhong, R.; Fowler, S.; Lyskowski, D.; Piyasena, H.; Carleton, K.; Spicer, C.; Ye, Z.H. The poplar MYB transcription factors, PtrMYB3 and PtrMYB20, are involved in the regulation of secondary wall biosynthesis. Plant Cell Physiol. 2010, 51, 1084-1090. [CrossRef]

50. Zhong, R.; Ye, Z.H. MYB46 and MYB83 bind to the SMRE sites and directly activate a suite of transcription factors and secondary wall biosynthetic genes. Plant Cell Physiol. 2012, 53, 368-380. [CrossRef]

51. Nakano, Y.; Yamaguchi, M.; Endo, H.; Rejab, N.A.; Ohtani, M. NAC-MYB-based transcriptional regulation of secondary cell wall biosynthesis in land plants. Front. Plant Sci. 2015, 6, 288. [CrossRef]

52. Ko, J.H.; Jeon, H.W.; Kim, W.C.; Kim, J.Y.; Han, K.H. The MYB46/MYB83-mediated transcriptional regulatory programme is a gatekeeper of secondary wall biosynthesis. Ann. Bot. 2014, 114, 1099-1107. [CrossRef]

53. Zhong, R.; Lee, C.; Ye, Z.H. Global analysis of direct targets of secondary wall NAC master switches in Arabidopsis. Mol. Plant 2010, 3, 1087-1103. [CrossRef] [PubMed]

54. Bollhöner, B.; Jokipii-Lukkari, S.; Bygdell, J.; Stael, S.; Adriasola, M.; Muñiz, L.; Van Breusegem, F.; Ezcurra, I.; Wingsle, G.; Tuominen, H. The function of two type II metacaspases in woody tissues of Populus trees. New Phytol. 2018, 217, 1551-1565. [CrossRef] [PubMed]

55. Endo, H.; Yamaguchi, M.; Tamura, T.; Nakano, Y.; Nishikubo, N.; Yoneda, A.; Kato, K.; Kubo, M.; Kajita, S.; Katayama, Y.; et al. Multiple classes of transcription factors regulate the expression of VASCULAR-RELATED NAC-DOMAIN7, a master switch of xylem vessel differentiation. Plant Cell Physiol. 2015, 56, 242-254. [CrossRef]

56. Wang, Y.W.; Wang, W.C.; Jin, S.H.; Wang, J.; Wang, B.; Hou, B.K. Over-expression of a putative poplar glycosyltransferase gene, PtGT1, in tobacco increases lignin content and causes early flowering. J. Exp. Bot. 2012, 63, 2799-2808. [CrossRef]

57. König, S.; Feussner, K.; Kaever, A.; Landesfeind, M.; Thurow, C.; Karlovsky, P.; Gatz, C.; Polle, A.; Feussner, I. Soluble phenylpropanoids are involved in the defense response of Arabidopsis against Verticillium longisporum. New Phytol. 2014, 202, 823-837. [CrossRef]

58. Rehman, H.M.; Nawaz, M.A.; Shah, Z.H.; Ludwig-Müller, J.; Chung, G.; Ahmad, M.Q.; Yang, S.H.; Lee, S.I. Comparative genomic and transcriptomic analyses of Family-1 UDP glycosyltransferase in three Brassica species and Arabidopsis indicates stress-responsive regulation. Sci. Rep. 2018, 8, 1875. [CrossRef]

59. Kosugi, S.; Hasebe, M.; Tomita, M.; Yanagawa, H. Systematic identification of cell cycle-dependent yeast nucleocytoplasmic shuttling proteins by prediction of composite motifs. Proc. Natl. Acad. Sci. USA 2009, 106, 10171-10176. [CrossRef] 
60. Wang, J.P.; Liu, B.; Sun, Y.; Chiang, V.L.; Sederoff, R.R. Enzyme-enzyme interactions in monolignol biosynthesis. Front. Plant Sci. 2019, 9, 1942. [CrossRef]

61. Bassard, J.E.; Richert, L.; Geerinck, J.; Renault, H.; Duval, F.; Ullmann, P.; Schmitt, M.; Meyer, E.; Mutterer, J.; Boerjan, W.; et al. Protein-protein and protein-membrane associations in the lignin pathway. Plant Cell 2012, 24, 4465-4482. [CrossRef]

62. Ono, N.N.; Qin, X.; Wilson, A.E.; Li, G.; Tian, L. Two UGT84 family glycosyltransferases catalyze a critical reaction of hydrolyzable tannin biosynthesis in pomegranate (Punica granatum). PLoS ONE 2016, 11, e0156319. [CrossRef]

63. Gallage, N.J.; Jørgensen, K.; Janfelt, C.; Nielsen, A.J.Z.; Naake, T.; Duński, E.; Dalsten, L.; Grisoni, M.; Møller, B.L. The intracellular localization of the vanillin biosynthetic machinery in pods of Vanilla planifolia. Plant Cell Physiol. 2018, 59, 304-318. [CrossRef]

64. Knudsen, C.; Gallage, N.J.; Hansen, C.C.; Møller, B.L.; Laursen, T. Dynamic metabolic solutions to the sessile life style of plants. Nat. Prod. Rep. 2018, 35, 1140-1155. [CrossRef]

65. Machettira, A.B.; Groß, L.E.; Tillmann, B.; Weis, B.L.; Englich, G.; Sommer, M.S.; Königer, M.; Schleiff, E. Protein-induced modulation of chloroplast membrane morphology. Front. Plant. Sci. 2012, 2. [CrossRef]

66. Perello, C.; Llamas, E.; Burlat, V.; Ortiz-Alcaide, M.; Phillips, M.A.; Pulido, P.; Rodriguez-Concepcion, M. Differential subplastidial localization and turnover of enzymes involved in isoprenoid biosynthesis in chloroplasts. PLoS ONE 2016, 11, e0150539. [CrossRef]

67. Armenteros, J.J.A.; Salvatore, M.; Emanuelsson, O.; Winther, O.; Von Heijne, G.; Elofsson, A.; Nielsen, H. Detecting sequence signals in targeting peptides using deep learning. Life Sci. Alliance 2019, 2. [CrossRef]

68. Baldacci-Cresp, F.; Moussawi, J.; Leplé, J.-C.; Van Acker, R.; Kohler, A.; Candiracci, J.; Twyffels, L.; Spokevicius, A.V.; Bossinger, G.; Laurans, F.; et al. PtaRHE1, a Populus tremula $\times$ Populus alba RING-H2 protein of the ATL family, has a regulatory role in secondary phloem fibre development. Plant. J. 2015, 82, 978-990. [CrossRef]

69. Liu, W.; Xie, Y.; Ma, J.; Luo, X.; Nie, P.; Zuo, Z.; Lahrmann, U.; Zhao, Q.; Zheng, Y.; Zhao, Y.; et al. IBS: An illustrator for the presentation and visualization of biological sequences. Bioinformatics 2015, 31, 3359-3361. [CrossRef]

70. Dereeper, A.; Guignon, V.; Blanc, G.; Audic, S.; Buffet, S.; Chevenet, F.; Dufayard, J.-F.; Guindon, S.; Lefort, V.; Lescot, M.; et al. Phylogeny.fr: Robust phylogenetic analysis for the non-specialist. Nucleic Acids Res. 2008, 36, W465-W469. [CrossRef]

71. Anisimova, M.; Gascuel, O. Approximate likelihood-ratio test for branches: A fast, accurate, and powerful alternative. Syst. Biol. 2006, 55, 539-552. [CrossRef]

72. Baldacci-Cresp, F.; Sacré, P.-Y.; Twyffels, L.; Mol, A.; Vermeersch, M.; Ziemons, E.; Hubert, P.; Pérez-Morga, D.; El Jaziri, M.; de Almeida Engler, J.; et al. Poplar-root knot nematode interaction: A model for perennial woody species. Mol. Plant.-Microbe Interact. 2016, 29, 560-572. [CrossRef]

73. Hemerly, A.S.; Ferreira, P.; de Almeida Engler, J.; Van Montagu, M.; Engler, G.; Inze, D. cdc2a expression in Arabidopsis is linked with competence for cell division. Plant. Cell 1993, 5, 1711-1723. [PubMed]

74. Karimi, M.; Inzé, D.; Depicker, A. GATEWAY ${ }^{\mathrm{TM}}$ vectors for Agrobacterium-mediated plant transformation. Trends Plant Sci. 2002, 7, 193-195. [CrossRef]

75. Nelson, B.K.; Cai, X.; Nebenführ, A. A multicolored set of in vivo organelle markers for co-localization studies in Arabidopsis and other plants. Plant J. 2007, 51, 1126-1136. [CrossRef] [PubMed]

76. Huang, F.C.; Giri, A.; Daniilidis, M.; Sun, G.; Härtl, K.; Hoffmann, T.; Schwab, W. Structural and functional analysis of UGT92G6 suggests an evolutionary link between mono- and disaccharide glycoside-forming transferases. Plant Cell Physiol. 2018, 59, 857-870. [CrossRef]

77. Leple, J.C.; Brasileiro, A.C.M.; Michel, M.F.; Delmotte, F.; Jouanin, L. Transgenic poplars: Expression of chimeric genes using four different constructs. Plant Cell Rep. 1992, 11, 137-141. [CrossRef] [PubMed] 
78. Van Acker, R.; Vanholme, R.; Storme, V.; Mortimer, J.; Dupree, P.; Boerjan, W. Lignin biosynthesis perturbations affect secondary cell wall composition and saccharification yield in Arabidopsis thaliana. Biotechnol. Biofuels 2013, 6, 46. [CrossRef]

79. Foster, C.E.; Martin, T.M.; Pauly, M. Comprehensive compositional analysis of plant cell walls (lignocellulosic biomass) Part I: Lignin. J. Vis. Exp. 2010, 37, e1745. [CrossRef] [PubMed]

(C) 2020 by the authors. Licensee MDPI, Basel, Switzerland. This article is an open access article distributed under the terms and conditions of the Creative Commons Attribution (CC BY) license (http://creativecommons.org/licenses/by/4.0/). 